\title{
Magnetizabilities of relativistic hydrogenlike atoms in some arbitrary discrete energy eigenstates
}

\author{
Patrycja Stefańska* \\ Atomic Physics Division, Department of Atomic, Molecular and Optical Physics, Faculty of Applied Physics and Mathematics, \\ Gdańsk University of Technology, Narutowicza 11/12, 80-233 Gdańsk, Poland
}

\section{Abstract}

We present the results of numerical calculations of magnetizability $(\chi)$ of the relativistic one-electron atoms with a pointlike, spinless and motionless nuclei of charge $Z e$. Exploiting the analytical formula for $\chi$ recently derived by us [P. Stefańska, 2015], valid for an arbitrary discrete energy eigenstate, we have found the values of the magnetizability for the ground state and for the first and the second set of excited states (i.e.: $2 s_{1 / 2}, 2 p_{1 / 2}, 2 p_{3 / 2}, 3 s_{1 / 2}, 3 p_{1 / 2}, 3 p_{3 / 2}, 3 d_{3 / 2}$, and $3 d_{5 / 2}$ ) of the Dirac one-electron atom. The results for ions with the atomic number $1 \leqslant Z \leqslant 137$ are given in 14 tables. The comparison of the numerical values of magnetizabilities for the ground state and for each states belonging to the first set of excited states of selected hydrogenlike ions, obtained with the use of two different values of the fine-structure 'constant, i.e.: $\alpha^{-1}=137.035999139$ (CODATA 2014) and $\alpha^{-1}=137.035999074$ (CODATA 2010), is also presented.

Keywords: Hydrogenlike atom, Magnetizability, Electromagnetic moments, Dipole moment, Magnetic field.

\section{Published as: At. Data Nucl. Data Tables 108 (2016) 193-210 doi: $10.1016 /$ j.adt.2015.09.001}

${ }^{*}$ Corresponding author.

Email address: pstefanska@mif.pg.gda.pl (Patrycja Stefańska) 


\section{Contents}

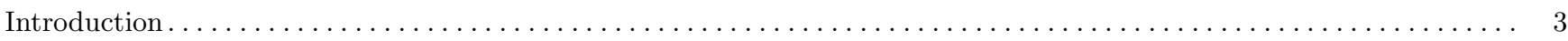

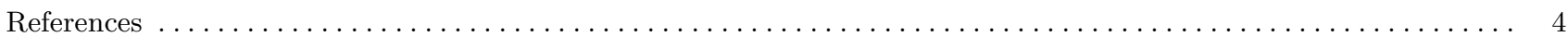

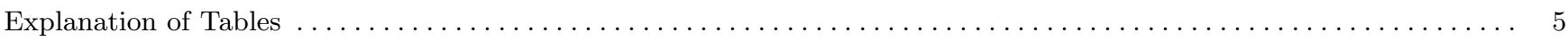

Tables

1. Relativistic magnetizabilities $\chi$ (in the units of $\left.\alpha^{2} a_{0}^{3}\right)$ for the ground state $\left(1 s_{1 / 2}\right)$ of hydrogenlike atoms, obtained with $\alpha^{-1}=137.035999139$ (CODATA 2014).

2. Relativistic magnetizabilities $\chi$ (in the units of $\alpha^{2} a_{0}^{3}$ ) of hydrogenlike atoms in the excited state $2 s_{1 / 2}$, obtained with $\alpha^{-1}=137.035999139$ (CODATA 2014).

3. Relativistic magnetizabilities $\chi$ (in the units of $\alpha^{2} a_{0}^{3}$ ) of hydrogenlike atoms in the excited state $2 p_{1 / 2}$, obtained with $\alpha^{-1}=137.035999139$ (CODATA 2014).

4. Relativistic magnetizabilities $\chi$ (in the units of $\alpha^{2} a_{0}^{3}$ ) of hydrogenlike atoms in the excited state $2 p_{3 / 2}(\mu= \pm 1 / 2)$, obtained with $\alpha^{-1}=137.035999139$ (CODATA 2014).

5. Relativistic magnetizabilities $\chi$ (in the units of $\alpha^{2} a_{0}^{3}$ ) of hydrogenlike atoms in the excited state $2 p_{3 / 2}(\mu= \pm 3 / 2$ ), obtained with $\alpha^{-1}=137.035999139$ (CODATA 2014)

6. Relativistic magnetizabilities $\chi$ (in the units of $\alpha^{2} a_{0}^{3}$ ) of hydrogenlike atoms in the excited state $3 s_{1 / 2}$, obtained with $\alpha^{-1}=137.035999139$ (CODATA 2014).

7. Relativistic magnetizabilities $\chi$ (in the units of $\alpha^{2} a_{0}^{3}$ ) of hydrogenlike atoms in the excited state $3 p_{1 / 2}$, obtained with $\alpha^{-1}=137.035999139$ (CODATA 2014).

8. Relativistic magnetizabilities $\chi$ (in the units of $\alpha^{2} a_{0}^{3}$ ) of hydrogenlike atoms in the excited state $3 p_{3 / 2}(\mu= \pm 1 / 2)$, obtained with $\alpha^{-1}=137.035999139$ (CODATA 2014).

9. Relativistic magnetizabilities $\chi$ (in the units of $\alpha^{2} a_{0}^{3}$ ) of hydrogenlike atoms in the excited state $3 p_{3 / 2}(\mu= \pm 3 / 2$ ), obtained with $\alpha^{-1}=137.035999139$ (CODATA 2014).

10. Relativistic magnetizabilities $\chi$ (in the units of $\alpha^{2} a_{0}^{3}$ ) of hydrogenlike atoms in the excited state $3 d_{3 / 2}(\mu= \pm 1 / 2$ ), obtained with $\alpha^{-1}=137.035999139$ (CODATA 2014).

Relativistic magnetizabilities $\chi$ (in the units of $\alpha^{2} a_{0}^{3}$ ) of hydrogenlike atoms in the excited state $3 d_{3 / 2}(\mu= \pm 3 / 2$ ), obtained with $\alpha^{-1}=137.035999139$ (CODATA 2014).

12. Relativistic magnetizabilities $\chi$ (in the units of $\alpha^{2} a_{0}^{3}$ ) of hydrogenlike atoms in the excited state $3 d_{5 / 2}(\mu= \pm 1 / 2)$, obtained with $\alpha^{-1}=137.035999139$ (CODATA 2014).

13. Relativistic magnetizabilities $\chi$ (in the units of $\alpha^{2} a_{0}^{3}$ ) of hydrogenlike atoms in the excited state $3 d_{5 / 2}(\mu= \pm 3 / 2)$, obtained with $\alpha^{-1}=137.035999139$ (CODATA 2014).

14. Relativistic magnetizabilities $\chi$ (in the units of $\alpha^{2} a_{0}^{3}$ ) of hydrogenlike atoms in the excited state $3 d_{5 / 2}(\mu= \pm 5 / 2)$, obtained with $\alpha^{-1}=137.035999139$ (CODATA 2014).

15. Relativistic magnetizabilities $\chi$ (in the units of $\alpha^{2} a_{0}^{3}$ ) for states $1 s_{1 / 2}, 2 s_{1 / 2}, 2 p_{1 / 2}$ and $2 p_{3 / 2}$ of selected hydrogenlike ions, obtained with $\alpha^{-1}=137.035999139$ (CODATA 2014) [the upper entries] and with $\alpha^{-1}=137.035999074$ (CODATA 2010) [the lower entries]. 


\section{Introduction}

Interaction of atoms and molecules with electromagnetic field is undoubtedly one of the most commonly reported physical processes, both theoretically and experimentally. For the simplest systems, like one-electron atoms, there are some analytical methods of calculating many atomic parameters, such as the polarizability or the magnetizability. One of such useful tool is the Sturmian expansion of the first-order generalized Dirac-Coulomb Green function [1], proposed by Szmytkowski in 1997. In the series of papers [2-9] published by his group over the period of past several years, it has been used in perturbation-theory calculations of some electromagnetic properties of the relativistic hydrogenlike atoms in the ground state, with a poinlike, spinless and motionless nuclei of charge $Z e$.

Recently, we have shown that the usefulness of this method goes beyond the study of the atomic ground state. In Ref. [10] we derived analytically an expression for the magnetizability of the Dirac one-electron atom (with regard to its nucleus we impose the same assumptions as above) in an arbitrary discrete energy eigenstate, characterized by the set of quantum numbers $\{n, \kappa, \mu\}$, in which $n$ denotes the radial quantum number, the Dirac quantum number $\kappa$ is an integer different form zero, whereas $\mu=-|\kappa|+\frac{1}{2},-|\kappa|+\frac{3}{2}, \ldots,|\kappa|-\frac{1}{2}$ is the magnetic quantum number. The final result has the following form:

$$
\begin{aligned}
\chi \equiv \chi_{n \kappa \mu}= & \frac{\alpha^{2} a_{0}^{3}}{Z^{2}} \frac{1}{128\left(4 \kappa^{2}-1\right)^{2} N_{n \kappa}}\left\{\Theta_{n \kappa \mu}^{(\mathrm{I})}+\sum_{\kappa^{\prime}} \frac{\eta_{\kappa \mu}^{(+)} \delta_{\kappa^{\prime},-\kappa+1}+\eta_{\kappa \mu}^{(-)} \delta_{\kappa^{\prime},-\kappa-1}}{N_{n \kappa}+\kappa^{\prime}}\right. \\
\times & {\left[\Theta_{n \kappa}^{(\mathrm{II})}+\frac{n !\left(n^{2}+2 n \gamma_{\kappa}+\kappa^{2}\right) \Gamma\left(n+2 \gamma_{\kappa}+1\right)}{\left(N_{n \kappa}-\kappa\right)\left(\gamma_{\kappa^{\prime}}-\gamma_{\kappa}-n+1\right) \Gamma\left(2 \gamma_{\kappa^{\prime}}+1\right)} \sum_{k=0}^{n} \sum_{p=0}^{n} \widetilde{\mathcal{Z}}_{\kappa \kappa^{\prime}}^{n}(k) \widetilde{\mathcal{Z}}_{\kappa \kappa^{\prime}}^{n}(p)\right.} \\
& \left.\left.\times{ }_{3} F_{2}\left(\begin{array}{c}
\gamma_{\kappa^{\prime}}-\gamma_{\kappa}-k-1, \gamma_{\kappa^{\prime}}-\gamma_{\kappa}-p-1, \gamma_{\kappa^{\prime}}-\gamma_{\kappa}-n+1 \\
\gamma_{\kappa^{\prime}}-\gamma_{\kappa}-n+2,2 \gamma_{\kappa^{\prime}}+1
\end{array}\right)\right]\right\},
\end{aligned}
$$

where $\alpha$ is the Sommerfeld's fine structure constant, $\Gamma(\zeta)$ denotes the Euler's gamma function, ${ }_{3} F_{2}$ is the generalized hypergeometric function, while

$$
\begin{gathered}
\eta_{\kappa \mu}^{( \pm)}=\left(4 \kappa^{2}-1\right)^{2}-4 \mu^{2}(2 \kappa \pm 1)^{2} \\
\Theta_{n \kappa \mu}^{(\mathrm{I})}=-256 \kappa^{2} \mu^{2}\left[2 \kappa^{2}\left(n+\gamma_{\kappa}\right)^{3}+\left(n+\gamma_{\kappa}\right)\left(5 n^{2}+10 n \gamma_{\kappa}+2 \gamma_{\kappa}^{2}-2 \kappa^{2}+1\right) N_{n \kappa}^{2}-\kappa\left(3 n^{2}+6 n \gamma_{\kappa}+4 \gamma_{\kappa}^{2}-\kappa^{2}\right) N_{n \kappa}\right], \\
\Theta_{n \kappa}^{(\mathrm{II})}=2\left(2 n+2 \gamma_{\kappa}+1\right)\left(\kappa-N_{n \kappa}\right) N_{n \kappa}^{2}\left[5\left(n+\gamma_{\kappa}\right)\left(n+\gamma_{\kappa}+1\right)-3\left(\gamma_{\kappa}^{2}-1\right)\right] \\
\widetilde{\mathcal{Z}}_{\kappa \kappa^{\prime}}^{n}(k)=\frac{(-)^{k}\left[2\left(N_{n \kappa}-\kappa\right)+(n-k)\left(\kappa+\kappa^{\prime}\right)\right]}{k !(n-k) !} \frac{\Gamma\left(\gamma_{\kappa}+\gamma_{\kappa^{\prime}}+k+2\right)}{\Gamma\left(k+2 \gamma_{\kappa}+1\right)}
\end{gathered}
$$

and analogously for $\widetilde{\mathcal{Z}}_{\kappa \kappa^{\prime}}^{n}(p)$, with

$$
N_{n \kappa}=\sqrt{n^{2}+2 n \gamma_{\kappa}+\kappa^{2}}
$$

and

$$
\gamma_{\kappa}=\sqrt{\kappa^{2}-(\alpha Z)^{2}}
$$

The above result has been exhaustively verified by us, both analytically and numerically. In Ref. [10] we have shown that it remains valid for an arbitrary discrete energy eigenstate. However, the aforementioned article contains only two representational tables with values of the relativistic magnetizabilities for some excited states of selected hydrogenlike ions. In this work, we present a more comprehensive numerical data in the form of 14 tables comprising the results for 
the atomic ground state $1 s_{1 / 2}$ and for each state belonging to the first and second set of excited states, i.e.: $2 s_{1 / 2}, 2 p_{1 / 2}$, $2 p_{3 / 2}, 3 s_{1 / 2}, 3 p_{1 / 2}, 3 p_{3 / 2}, 3 d_{3 / 2}$ and $3 d_{5 / 2}$, having regard all possible values of the magnetic quantum number.

Present calculations have been performed with the current value 137.035999139 of the inverse of the fine-structure constant recommended by the Committee on Data for Science and Technology (CODATA) [11], in contrast to those described in Ref. [10], in which we have used $\alpha^{-1}=137.0359895$ (CODATA 1986), to be able to compare our results with the previous results of other authors [12]. To show how the change in the value of the fine structure constant affects the value of the magnetizability, additionally, we performed calculations for $\chi$ with $\alpha^{-1}=137.035999074$, recommended by CODATA 2010 (i.e. immediately before the currently valid value of this constant) and compared them with the corresponding values from Tables $1-5$. The appropriate juxtaposition of numerical values of $\chi$ for the ground state and the first excited states of selected hydrogenlike ions are shown in Table 15.

\section{Acknowledgments}

I wish to thank Professor R. Szmytkowski for many stimulating discussions, especially for every advice he gave me during the preparation of this work.

\section{References}

\section{References}

[1] R. Szmytkowski, Journal of Physics B 30 (1997) 825 [erratum: Journal of Physics B 30 (1997) 2747; addendum: arXiv:physics/9902050].

[2] R. Szmytkowski, Journal of Physics B 35 (2002) 1379.

[3] R. Szmytkowski, K. Mielewczyk, Journal of Physics B 37 (2004) 3961.

[4] R. Szmytkowski, Physical Review A 65 (2002) 012503.

[5] K. Mielewczyk, R. Szmytkowski, Physical Review A 73 (2006) 022511 [erratum: Physical Review A 73 (2006) 039908].

[6] R. Szmytkowski, P. Stefańska, e-print arXiv:1102.1811.

[7] P. Stefańska, R. Szmytkowski, International Journal of Quantum Chemistry 112 (2012) 1363.

[8] R. Szmytkowski, P. Stefańska, Physical Review A 85 (2012) 042502.

[9] R. Szmytkowski and P. Stefańska, Physical Review A 89 (2014) 012501.

[10] P. Stefańska, Physical Review A 92 (2015) 032504.

[11] CODATA Internationally recommended 2014 values of the fundamental physical constants, http://physics.nist.gov/cuu/Constants/index.html.

[12] A. Rutkowski, A. Poszwa, Physical Review A 75 (2007) 033402. 


\section{Explanation of Tables}

In all the tables we have used the following notation:

$\chi$

$Z$

$\alpha$

$a_{0}$

$a(b)$

$\mu$ the atomic magnetizability

the atomic number

the fine structure constant

the Bohr radius

this means $a \times 10^{b}$

the magnetic quantum number; $\mu=-j, \ldots, j$

The atomic states are referred to as $\mathcal{N} x_{j}$, due to the following guidelines:

$\mathcal{N}$ the principal quantum number

$\mathcal{N}=n+|\kappa| \quad$ Note that for the radial quantum number $n=0, \kappa$ is the negative integer! $\kappa$ the Dirac quantum number; $\kappa=(l-j)(2 j+1)$

$j \quad$ the total angular momentum quantum number; $j=|\kappa|-\frac{1}{2}=l \pm \frac{1}{2}$

$x \equiv s \quad$ states with the orbital angular momentum quantum number $l=0$

$x \equiv p \quad$ states with the orbital angular momentum quantum number $l=1$

$x \equiv d \quad$ states with the orbital angular momentum quantum number $l=2$ 
Table 1

Relativistic magnetizabilities $\chi$ (in the units of $\alpha^{2} a_{0}^{3}$ ) for the ground state $\left(1 s_{1 / 2}\right)$ of hydrogenlike atoms, obtained with $\alpha^{-1}=137.035999139$ (CODATA 2014).

\begin{tabular}{|c|c|c|c|c|c|}
\hline$Z$ & $\chi\left[\alpha^{2} a_{0}^{3}\right]$ & $Z$ & $\chi\left[\alpha^{2} a_{0}^{3}\right]$ & $Z$ & $\chi\left[\alpha^{2} a_{0}^{3}\right]$ \\
\hline 1 & $-4.999644993669(-1)$ & 47 & $-1.914539761522(-4)$ & 93 & $-2.485921010714(-5)$ \\
\hline 2 & $-1.249645001764(-1)$ & 48 & $-1.821478207432(-4)$ & 94 & $-2.369686853502(-5)$ \\
\hline 3 & $-5.552005708136(-2)$ & 49 & $-1.734078279628(-4)$ & 95 & $-2.257394374192(-5)$ \\
\hline 4 & $-3.121450341512(-2)$ & 50 & $-1.651892129562(-4)$ & 96 & $-2.148886006463(-5)$ \\
\hline 5 & $-1.996450584458(-2)$ & 51 & $-1.574515385043(-4)$ & 97 & $-2.044012432389(-5)$ \\
\hline 6 & $-1.385339770334(-2)$ & 52 & $-1.501582182786(-4)$ & 98 & $-1.942632090285(-5)$ \\
\hline 7 & $-1.016859395768(-2)$ & 53 & $-1.432760850756(-4)$ & 99 & $-1.844610718053(-5)$ \\
\hline 8 & $-7.777016376682(-3)$ & 54 & $-1.367750144935(-4)$ & 100 & $-1.749820929318(-5)$ \\
\hline 9 & $-6.137360476020(-3)$ & 55 & $-1.306275960639(-4)$ & 101 & $-1.658141819878(-5)$ \\
\hline 10 & $-4.964526105003(-3)$ & 56 & $-1.248088451191(-4)$ & 102 & $-1.569458602238(-5)$ \\
\hline 11 & $-4.096763187651(-3)$ & 57 & $-1.192959497297(-4)$ & 103 & $-1.483662266206(-5)$ \\
\hline 12 & $-3.436760225738(-3)$ & 58 & $-1.140680479176(-4)$ & 104 & $-1.400649263726(-5)$ \\
\hline 13 & $-2.923124649789(-3)$ & 59 & $-1.091060310748(-4)$ & 105 & $-1.320321216323(-5)$ \\
\hline 14 & $-2.515572485422(-3)$ & 60 & $-1.043923701231(-4)$ & 106 & $-1.242584643679(-5)$ \\
\hline 15 & $-2.186782153895(-3)$ & 61 & $-9.991096145807(-5)$ & 107 & $-1.167350712035(-5)$ \\
\hline 16 & $-1.917693332212(-3)$ & 62 & $-9.564699014466(-5)$ & 108 & $-1.094535001293(-5)$ \\
\hline 17 & $-1.694681086002(-3)$ & 63 & $-9.158680819165(-5)$ & 109 & $-1.024057289792(-5)$ \\
\hline 18 & $-1.507796651860(-3)$ & 64 & $-8.771782603565(-5)$ & 110 & $-9.558413559454(-6)$ \\
\hline 19 & $-1.349638371108(-3)$ & 65 & $-8.402841562093(-5)$ & 111 & $-8.898147960202(-6)$ \\
\hline 20 & $-1.214607414490(-3)$ & 66 & $-8.050782368085(-5)$ & 112 & $-8.259088575604(-6)$ \\
\hline 21 & $-1.098405408420(-3)$ & 67 & $-7.714609401184(-5)$ & 113 & $-7.640582880810(-6)$ \\
\hline 22 & $-9.976881098837(-4)$ & 68 & $-7.393399769008(-5)$ & 114 & $-7.042011988848(-6)$ \\
\hline 23 & $-9.098220947731(-4)$ & 69 & $-7.086297031681(-5)$ & 115 & $-6.462789440666(-6)$ \\
\hline 24 & $-8.327108732660(-4)$ & 70 & $-6.792505549481(-5)$ & 116 & $-5.902360150332(-6)$ \\
\hline 25 & $-7.646 \quad 686812239(-4)$ & 71 & $-6.511285383916(-5)$ & 117 & $-5.360199511955(-6)$ \\
\hline 26 & $-7.043275730781(-4)$ & 72 & $-6.241947691165(-5)$ & 118 & $-4.835812678917(-6)$ \\
\hline 27 & $-6.505681398115(-4)$ & 73 & $-5.983850554327(-5)$ & 119 & $-4.328734031249(-6)$ \\
\hline 28 & $-6.024672275918(-4)$ & 74 & $-5.736395207348(-5)$ & 120 & $-3.838526853900(-6)$ \\
\hline 29 & $-5.592580513314(-4)$ & 75 & $-5.499022609171(-5)$ & 121 & $-3.364783257947(-6)$ \\
\hline 30 & $-5.202994550774(-4)$ & 76 & $-5.271210331468(-5)$ & 122 & $-2.907124389732(-6)$ \\
\hline 31 & $-4.850519982467(-4)$ & 77 & $-5.052469727622(-5)$ & 123 & $-2.465200991082(-6)$ \\
\hline 32 & $-4.530591888651(-4)$ & 78 & $-4.842343354310(-5)$ & 124 & $-2.038694400207(-6)$ \\
\hline 33 & $-4.239326356104(-4)$ & 79 & $-4.640402620272(-5)$ & 125 & $-1.627318121987(-6)$ \\
\hline 34 & $-3.973402106035(-4)$ & 80 & $-4.446245639716(-5)$ & 126 & $-1.230820156192(-6)$ \\
\hline 35 & $-3.729965449227(-4)$ & 81 & $-4.259495270292(-5)$ & 127 & $-8.489863663198(-7)$ \\
\hline 36 & $-3.506553458869(-4)$ & 82 & $-4.079797317743(-5)$ & 128 & $-4.816453253524(-7)$ \\
\hline 37 & $-3.301031476936(-4)$ & 83 & $-3.906818891304(-5)$ & 129 & $-1.286753358938(-7)$ \\
\hline 38 & $-3.111541977427(-4)$ & 84 & $-3.740246895575(-5)$ & 130 & $2.099852117594(-7)$ \\
\hline 39 & $-2.936462487550(-4)$ & 85 & $-3.579786646142(-5)$ & 131 & $5.343220977306(-7)$ \\
\hline 40 & $-2.774370778562(-4)$ & 86 & $-3.425160597514(-5)$ & 132 & $8.442203047896(-7)$ \\
\hline 41 & $-2.624015925544(-4)$ & 87 & $-3.276107173135(-5)$ & 133 & $1.139421970186(-6)$ \\
\hline 42 & $-2.484294131889(-4)$ & 88 & $-3.132379688278(-5)$ & 134 & $1.419449261336(-6)$ \\
\hline 43 & $-2.354228442610(-4)$ & 89 & $-2.993745357549(-5)$ & 135 & $1.683443662506(-6)$ \\
\hline 44 & $-2.232951647654(-4)$ & 90 & $-2.859984379546(-5)$ & 136 & $1.929752760437(-6)$ \\
\hline 45 & $-2.119691814557(-4)$ & 91 & $-2.730889091984(-5)$ & 137 & $2.154582953028(-6)$ \\
\hline 46 & $-2.013759998257(-4)$ & 92 & $-2.606263191219(-5)$ & & \\
\hline
\end{tabular}


Table 2

Relativistic magnetizabilities $\chi$ (in the units of $\alpha^{2} a_{0}^{3}$ ) of hydrogenlike atoms in the excited state $2 s_{1 / 2}$, obtained with $\alpha^{-1}=137.035999139$ (CODATA 2014).

\begin{tabular}{|c|c|c|c|c|c|}
\hline$Z$ & $\chi\left[\alpha^{2} a_{0}^{3}\right]$ & $Z$ & $\chi\left[\alpha^{2} a_{0}^{3}\right]$ & $Z$ & $\chi\left[\alpha^{2} a_{0}^{3}\right]$ \\
\hline 1 & $-6.999722649147(+0)$ & 47 & $-2.891300079244(-3)$ & 93 & $-5.295132413795(-4)$ \\
\hline 2 & $-1.749722649002(+0)$ & 48 & $-2.760626290162(-3)$ & 94 & $-5.122608926758(-4)$ \\
\hline 3 & $-7.775004265370(-1)$ & 49 & $-2.637869458376(-3)$ & 95 & $-4.955404845206(-4)$ \\
\hline 4 & $-4.372226484179(-1)$ & 50 & $-2.522402512753(-3)$ & 96 & $-4.793293766449(-4)$ \\
\hline 5 & $-2.797226479764(-1)$ & 51 & $-2.413659246069(-3)$ & 97 & $-4.636060481284(-4)$ \\
\hline 6 & $-1.941670918773(-1)$ & 52 & $-2.311127360473(-3)$ & 98 & $-4.483500254287(-4)$ \\
\hline 7 & $-1.425797896419(-1)$ & 53 & $-2.214342422647(-3)$ & 99 & $-4.335418150281(-4)$ \\
\hline 8 & $-1.090976460292(-1)$ & 54 & $-2.122882595177(-3)$ & 100 & $-4.191628402646(-4)$ \\
\hline 9 & $-8.614239824945(-2)$ & 55 & $-2.036364032257(-3)$ & 101 & $-4.051953819423(-4)$ \\
\hline 10 & $-6.972264418235(-2)$ & 56 & $-1.954436845705(-3)$ & 102 & $-3.916225223358(-4)$ \\
\hline 11 & $-5.757388275239(-2)$ & 57 & $-1.876781561951(-3)$ & 103 & $-3.784280922233(-4)$ \\
\hline 12 & $-4.833375297132(-2)$ & 58 & $-1.803106002867(-3)$ & 104 & $-3.655966205901(-4)$ \\
\hline 13 & $-4.114275885212(-2)$ & 59 & $-1.733142533473(-3)$ & 105 & $-3.531132866548(-4)$ \\
\hline 14 & $-3.543692473776(-2)$ & 60 & $-1.666645628015(-3)$ & 106 & $-3.409638738663(-4)$ \\
\hline 15 & $-3.083374850888(-2)$ & 61 & $-1.603389712992(-3)$ & 107 & $-3.291347255127(-4)$ \\
\hline 16 & $-2.706638562519(-2)$ & 62 & $-1.543167251719(-3)$ & 108 & $-3.176127015690(-4)$ \\
\hline 17 & $-2.394408698585(-2)$ & 63 & $-1.485787039964(-3)$ & 109 & $-3.063851363791(-4)$ \\
\hline 18 & $-2.132756988218(-2)$ & 64 & $-1.431072686523(-3)$ & 110 & $-2.954397967327(-4)$ \\
\hline 19 & $-1.911321107028(-2)$ & 65 & $-1.378861256103(-3)$ & 111 & $-2.847648398359(-4)$ \\
\hline 20 & $-1.722262691678(-2)$ & 66 & $-1.329002055036(-3)$ & 112 & $-2.743487706001(-4)$ \\
\hline 21 & $-1.559564016628(-2)$ & 67 & $-1.281355542857(-3)$ & 113 & $-2.641803975620(-4)$ \\
\hline 22 & $-1.418543138991(-2)$ & 68 & $-1.235792355067(-3)$ & 114 & $-2.542487866056(-4)$ \\
\hline 23 & $-1.295513262209(-2)$ & 69 & $-1.192192424289(-3)$ & 115 & $-2.445432114536(-4)$ \\
\hline 24 & $-1.187539297571(-2)$ & 70 & $-1.150444188624(-3)$ & 116 & $-2.350530996246(-4)$ \\
\hline 25 & $-1.092261172173(-2)$ & 71 & $-1.110443877486(-3)$ & 117 & $-2.257679721772(-4)$ \\
\hline 26 & $-1.007763758957(-2)$ & 72 & $-1.072094866329(-3)$ & 118 & $-2.166773750318(-4)$ \\
\hline 27 & $-9.324798818781(-3)$ & 73 & $-1.035307092789(-3)$ & 119 & $-2.077707989250(-4)$ \\
\hline 28 & $-8.651171219921(-3)$ & 74 & $-9.999965276428(-4)$ & 120 & $-1.990375839852(-4)$ \\
\hline 29 & $-8.046019764341(-3)$ & 75 & $-9.660846947558(-4)$ & 121 & $-1.904668033712(-4)$ \\
\hline 30 & $-7.500368229614(-3)$ & 76 & $-9.334982349096(-4)$ & 122 & $-1.820471181228(-4)$ \\
\hline 31 & $-7.006664406792(-3)$ & 77 & $-9.021685089679(-4)$ & 123 & $-1.737665918852(-4)$ \\
\hline 32 & $-6.558517365714(-3)$ & 78 & $-8.720312363693(-4)$ & 124 & $-1.656124487687(-4)$ \\
\hline 33 & $-6.150489583572(-3)$ & 79 & $-8.430261653873(-4)$ & 125 & $-1.575707489732(-4)$ \\
\hline 34 & $-5.777931223941(-3)$ & 80 & $-8.150967719944(-4)$ & 126 & $-1.496259425995(-4)$ \\
\hline 35 & $-5.436847073938(-3)$ & 81 & $-7.881899845170(-4)$ & 127 & $-1.417602378199(-4)$ \\
\hline 36 & $-5.123788986136(-3)$ & 82 & $-7.622559315742(-4)$ & 128 & $-1.339526764241(-4)$ \\
\hline 37 & $-4.835768387453(-3)$ & 83 & $-7.372477110602(-4)$ & 129 & $-1.261777290721(-4)$ \\
\hline 38 & $-4.570184687625(-3)$ & 84 & $-7.131211781713(-4)$ & 130 & $-1.184030625960(-4)$ \\
\hline 39 & $-4.324766368820(-3)$ & 85 & $-6.898347506852(-4)$ & 131 & $-1.105857907160(-4)$ \\
\hline 40 & $-4.097522252752(-3)$ & 86 & $-6.673492298868(-4)$ & 132 & $-1.026657241129(-4)$ \\
\hline 41 & $-3.886700984303(-3)$ & 87 & $-6.456276356981(-4)$ & 133 & $-9.455204905699(-5)$ \\
\hline 42 & $-3.690757185747(-3)$ & 88 & $-6.246350547147(-4)$ & 134 & $-8.609343611784(-5)$ \\
\hline 43 & $-3.508323055315(-3)$ & 89 & $-6.043384999803(-4)$ & 135 & $-7.699646244933(-5)$ \\
\hline 44 & $-3.338184431761(-3)$ & 90 & $-5.847067814448(-4)$ & 136 & $-6.650881273142(-5)$ \\
\hline 45 & $-3.179260540006(-3)$ & 91 & $-5.657103861534(-4)$ & 137 & $-4.903302713912(-5)$ \\
\hline 46 & $-3.030586784802(-3)$ & 92 & $-5.473213673024(-4)$ & & \\
\hline
\end{tabular}


Table 3

Relativistic magnetizabilities $\chi$ (in the units of $\alpha^{2} a_{0}^{3}$ ) of hydrogenlike atoms in the excited state $2 p_{1 / 2}$, obtained with $\alpha^{-1}=137.035999139$ (CODATA 2014).

\begin{tabular}{|c|c|c|c|c|c|}
\hline$Z$ & $\chi\left[\alpha^{2} a_{0}^{3}\right]$ & $Z$ & $\chi\left[\alpha^{2} a_{0}^{3}\right]$ & $Z$ & $\chi\left[\alpha^{2} a_{0}^{3}\right]$ \\
\hline 1 & $6.676163152752(+4)$ & 47 & $1.041492649963(-2)$ & 93 & $2.075091052946(-4)$ \\
\hline 2 & $4.171164660772(+3)$ & 48 & $9.452888853908(-3)$ & 94 & $1.888736138669(-4)$ \\
\hline 3 & $8.234606720151(+2)$ & 49 & $8.591529248763(-3)$ & 95 & $1.715865142938(-4)$ \\
\hline 4 & $2.603386065977(+2)$ & 50 & $7.818803550735(-3)$ & 96 & $1.555531822422(-4)$ \\
\hline 5 & $1.065244131032(+2)$ & 51 & $7.124297098369(-3)$ & 97 & $1.406862128024(-4)$ \\
\hline 6 & $5.130676007305(+1)$ & 52 & $6.498978907081(-3)$ & 98 & $1.269048169139(-4)$ \\
\hline 7 & $2.765269415830(+1)$ & 53 & $5.934996687137(-3)$ & 99 & $1.141342734163(-4)$ \\
\hline 8 & $1.618150944960(+1)$ & 54 & $5.425505295092(-3)$ & 100 & $1.023054311643(-4)$ \\
\hline 9 & $1.008224808325(+1)$ & 55 & $4.964522679064(-3)$ & 101 & $9.135425625123(-5)$ \\
\hline 10 & $6.600466097095(+0)$ & 56 & $4.546808517220(-3)$ & 102 & $8.122141992195(-5)$ \\
\hline 11 & $4.497274971042(+0)$ & 57 & $4.167761653933(-3)$ & 103 & $7.185192323468(-5)$ \\
\hline 12 & $3.166932216060(+0)$ & 58 & $3.823333160207(-3)$ & 104 & $6.319475495001(-5)$ \\
\hline 13 & $2.292616774375(+0)$ & 59 & $3.509952423720(-3)$ & 105 & $5.520257950219(-5)$ \\
\hline 14 & $1.699146904160(+0)$ & 60 & $3.224464139363(-3)$ & 106 & $4.783145224150(-5)$ \\
\hline 15 & $1.285028571997(+0)$ & 61 & $2.964074447113(-3)$ & 107 & $4.104055943473(-5)$ \\
\hline 16 & $9.890736419919(-1)$ & 62 & $2.726304768887(-3)$ & 108 & $3.479198077905(-5)$ \\
\hline 17 & $7.731059146983(-1)$ & 63 & $2.508952143920(-3)$ & 109 & $2.905047242532(-5)$ \\
\hline 18 & $6.125838134843(-1)$ & 64 & $2.310055064618(-3)$ & 110 & $2.378326872591(-5)$ \\
\hline 19 & $4.913104810651(-1)$ & 65 & $2.127863980587(-3)$ & 111 & $1.895990112171(-5)$ \\
\hline 20 & $3.983443339545(-1)$ & 66 & $1.960815774794(-3)$ & 112 & $1.455203276815(-5)$ \\
\hline 21 & $3.261385629899(-1)$ & 67 & $1.807511628042(-3)$ & 113 & $1.053330767350(-5)$ \\
\hline 22 & $2.693897023870(-1)$ & 68 & $1.666697780826(-3)$ & 114 & $6.879213289411(-6)$ \\
\hline 23 & $2.243073696744(-1)$ & 69 & $1.537248778577(-3)$ & 115 & $3.566955658159(-6)$ \\
\hline 24 & $1.881407837991(-1)$ & 70 & $1.418152850346(-3)$ & 116 & $5.753463894641(-7)$ \\
\hline 25 & $1.588655676583(-1)$ & 71 & $1.308499124331(-3)$ & 117 & $-2.115299080229(-6)$ \\
\hline 26 & $1.349727657448(-1)$ & 72 & $1.207466428302(-3)$ & 118 & $-4.523252290108(-6)$ \\
\hline 27 & $1.153243297378(-1)$ & 73 & $1.114313460394(-3)$ & 119 & $-6.665451722083(-6)$ \\
\hline 28 & $9.905260581612(-2)$ & 74 & $1.028370147165(-3)$ & 120 & $-8.557571732907(-6)$ \\
\hline 29 & $8.548943353449(-2)$ & 75 & $9.490300323220(-4)$ & 121 & $-1.021407985150(-5)$ \\
\hline 30 & $7.411547649680(-2)$ & 76 & $8.757435618780(-4)$ & 122 & $-1.164828128722(-5)$ \\
\hline 31 & $6.452357143356(-2)$ & 77 & $8.080121504470(-4)$ & 123 & $-1.287234869347(-5)$ \\
\hline 32 & $5.639191775860(-2)$ & 78 & $7.453829294325(-4)$ & 124 & $-1.389733402654(-5)$ \\
\hline 33 & $4.946425903654(-2)$ & 79 & $6.874440915371(-4)$ & 125 & $-1.473315745991(-5)$ \\
\hline 34 & $4.353508886238(-2)$ & 80 & $6.338207576445(-4)$ & 126 & $-1.538856526852(-5)$ \\
\hline 35 & $3.843850568001(-2)$ & 81 & $5.841713020641(-4)$ & 127 & $-1.587104346068(-5)$ \\
\hline 36 & $3.403974400708(-2)$ & 82 & $5.381840806272(-4)$ & 128 & $-1.618666488046(-5)$ \\
\hline 37 & $3.022868709620(-2)$ & 83 & $4.955745134059(-4)$ & 129 & $-1.633983071444(-5)$ \\
\hline 38 & $2.691485942864(-2)$ & 84 & $4.560824800844(-4)$ & 130 & $-1.633283429506(-5)$ \\
\hline 39 & $2.402353358305(-2)$ & 85 & $4.194699913940(-4)$ & 131 & $-1.616510518910(-5)$ \\
\hline 40 & $2.149268284498(-2)$ & 86 & $3.855191046650(-4)$ & 132 & $-1.583182968338(-5)$ \\
\hline 41 & $1.927058043264(-2)$ & 87 & $3.540300555545(-4)$ & 133 & $-1.532122229934(-5)$ \\
\hline 42 & $1.731389656364(-2)$ & 88 & $3.248195814763(-4)$ & 134 & $-1.460843494070(-5)$ \\
\hline 43 & $1.558618136759(-2)$ & 89 & $2.977194152621(-4)$ & 135 & $-1.363909968444(-5)$ \\
\hline 44 & $1.405664873271(-2)$ & 90 & $2.725749301911(-4)$ & 136 & $-1.226629949283(-5)$ \\
\hline 45 & $1.269919627128(-2)$ & 91 & $2.492439197909(-4)$ & 137 & $-9.398566990645(-6)$ \\
\hline 46 & $1.149161160811(-2)$ & 92 & $2.275954977863(-4)$ & & \\
\hline
\end{tabular}


Table 4

Relativistic magnetizabilities $\chi$ (in the units of $\left.\alpha^{2} a_{0}^{3}\right)$ of hydrogenlike atoms in the excited state $2 p_{3 / 2}(\mu= \pm 1 / 2)$, obtained with $\alpha^{-1}=$ 137.035999139 (CODATA 2014).

\begin{tabular}{|c|c|c|c|c|c|}
\hline$Z$ & $\chi\left[\alpha^{2} a_{0}^{3}\right]$ & $Z$ & $\chi\left[\alpha^{2} a_{0}^{3}\right]$ & $Z$ & $\chi\left[\alpha^{2} a_{0}^{3}\right]$ \\
\hline 1 & $-6.677063123774(+4)$ & 47 & $-1.419858008271(-2)$ & 93 & $-9.520247585051(-4)$ \\
\hline 2 & $-4.173414370995(+3)$ & 48 & $-1.306850716417(-2)$ & 94 & $-9.110967976025(-4)$ \\
\hline 3 & $-8.244603822364(+2)$ & 49 & $-1.204929044477(-2)$ & 95 & $-8.721966496674(-4)$ \\
\hline 4 & $-2.609008168169(+2)$ & 50 & $-1.112807953802(-2)$ & 96 & $-8.352003098775(-4)$ \\
\hline 5 & $-1.068841233198(+2)$ & 51 & $-1.029373178218(-2)$ & 97 & $-7.999924087903(-4)$ \\
\hline 6 & $-5.155647028648(+1)$ & 52 & $-9.536557503502(-3)$ & 98 & $-7.664655136787(-4)$ \\
\hline 7 & $-2.783607783733(+1)$ & 53 & $-8.848107262219(-3)$ & 99 & $-7.345194910790(-4)$ \\
\hline 8 & $-1.632184465485(+1)$ & 54 & $-8.220993530005(-3)$ & 100 & $-7.040609243754(-4)$ \\
\hline 9 & $-1.019306939462(+1)$ & 55 & $-7.648740715368(-3)$ & 101 & $-6.750025808723(-4)$ \\
\hline 10 & $-6.690176291750(+0)$ & 56 & $-7.125658615537(-3)$ & 102 & $-6.472629233627(-4)$ \\
\hline 11 & $-4.571365324767(+0)$ & 57 & $-6.646735297241(-3)$ & 103 & $-6.207656616747(-4)$ \\
\hline 12 & $-3.229142397650(+0)$ & 58 & $-6.207546146715(-3)$ & 104 & $-5.954393400982(-4)$ \\
\hline 13 & $-2.345581386355(+0)$ & 59 & $-5.804176421013(-3)$ & 105 & $-5.712169569462(-4)$ \\
\hline 14 & $-1.744775437492(+0)$ & 60 & $-5.433155109121(-3)$ & 106 & $-5.480356128082(-4)$ \\
\hline 15 & $-1.324738729201(+0)$ & 61 & $-5.091398296516(-3)$ & 107 & $-5.258361842946(-4)$ \\
\hline 16 & $-1.023940039748(+0)$ & 62 & $-4.776160539216(-3)$ & 108 & $-5.045630202691(-4)$ \\
\hline 17 & $-8.039579208388(-1)$ & 63 & $-4.484993007746(-3)$ & 109 & $-4.841636576980(-4)$ \\
\hline 18 & $-6.400717180687(-1)$ & 64 & $-4.215707369325(-3)$ & 110 & $-4.645885543258(-4)$ \\
\hline 19 & $-5.159513442642(-1)$ & 65 & $-3.966344546946(-3)$ & 111 & $-4.457908353960(-4)$ \\
\hline 20 & $-4.205544369778(-1)$ & 66 & $-3.735147634167(-3)$ & 112 & $-4.277260515650(-4)$ \\
\hline 21 & $-3.462568162852(-1)$ & 67 & $-3.520538360073(-3)$ & 113 & $-4.103519449886(-4)$ \\
\hline 22 & $-2.876948199888(-1)$ & 68 & $-3.321096594543(-3)$ & 114 & $-3.936282202669(-4)$ \\
\hline 23 & $-2.410306639419(-1)$ & 69 & $-3.135542463400(-3)$ & 115 & $-3.775163164680(-4)$ \\
\hline 24 & $-2.034758302313(-1)$ & 70 & $-2.962720709108(-3)$ & 116 & $-3.619791757628(-4)$ \\
\hline 25 & $-1.729755979538(-1)$ & 71 & $-2.801586987902(-3)$ & 117 & $-3.469810031876(-4)$ \\
\hline 26 & $-1.479963885340(-1)$ & 72 & $-2.651195840403(-3)$ & 118 & $-3.324870105890(-4)$ \\
\hline 27 & $-1.273800042385(-1)$ & 73 & $-2.510690111521(-3)$ & 119 & $-3.184631356651(-4)$ \\
\hline 28 & $-1.102421744243(-1)$ & 74 & $-2.379291628090(-3)$ & 120 & $-3.048757238860(-4)$ \\
\hline 29 & $-9.590093645933(-2)$ & 75 & $-2.256292970148(-3)$ & 121 & $-2.916911564274(-4)$ \\
\hline 30 & $-8.382541308263(-2)$ & 76 & $-2.141050195036(-3)$ & 122 & $-2.788754002214(-4)$ \\
\hline 31 & $-7.359873103395(-2)$ & 77 & $-2.032976393189(-3)$ & 123 & $-2.663934454106(-4)$ \\
\hline 32 & $-6.489087280871(-2)$ & 78 & $-1.931535971212(-3)$ & 124 & $-2.542085784218(-4)$ \\
\hline 33 & $-5.743859086216(-2)$ & 79 & $-1.836239572101(-3)$ & 125 & $-2.422814112148(-4)$ \\
\hline 34 & $-5.103040041916(-2)$ & 80 & $-1.746639554569(-3)$ & 126 & $-2.305685410031(-4)$ \\
\hline 35 & $-4.549526321186(-2)$ & 81 & $-1.662325963868(-3)$ & 127 & $-2.190206345130(-4)$ \\
\hline 36 & $-4.069398041558(-2)$ & 82 & $-1.582922935355(-3)$ & 128 & $-2.075795856830(-4)$ \\
\hline 37 & $-3.651259282147(-2)$ & 83 & $-1.508085479692(-3)$ & 129 & $-1.961741195534(-4)$ \\
\hline 38 & $-3.285728128388(-2)$ & 84 & $-1.437496605132(-3)$ & 130 & $-1.847126573331(-4)$ \\
\hline 39 & $-2.965039784717(-2)$ & 85 & $-1.370864738001(-3)$ & 131 & $-1.730710455245(-4)$ \\
\hline 40 & $-2.682735569862(-2)$ & 86 & $-1.307921407350(-3)$ & 132 & $-1.610698646631(-4)$ \\
\hline 41 & $-2.433417630171(-2)$ & 87 & $-1.248419163964(-3)$ & 133 & $-1.484282849689(-4)$ \\
\hline 42 & $-2.212554294681(-2)$ & 88 & $-1.192129707603(-3)$ & 134 & $-1.346569662465(-4)$ \\
\hline 43 & $-2.016324714763(-2)$ & 89 & $-1.138842199469(-3)$ & 135 & $-1.187541215741(-4)$ \\
\hline 44 & $-1.841494171369(-2)$ & 90 & $-1.088361739674(-3)$ & 136 & $-9.796700216327(-5)$ \\
\hline 45 & $-1.685313467441(-2)$ & 91 & $-1.040507991880(-3)$ & 137 & $-4.903236487356(-5)$ \\
\hline 46 & $-1.545437344510(-2)$ & 92 & $-9.951139393523(-4)$ & & \\
\hline
\end{tabular}


Table 5

Relativistic magnetizabilities $\chi$ (in the units of $\left.\alpha^{2} a_{0}^{3}\right)$ of hydrogenlike atoms in the excited state $2 p_{3 / 2}(\mu= \pm 3 / 2)$, obtained with $\alpha^{-1}=$ 137.035999139 (CODATA 2014).

\begin{tabular}{|c|c|c|c|c|c|}
\hline$Z$ & $\chi\left[\alpha^{2} a_{0}^{3}\right]$ & $Z$ & $\chi\left[\alpha^{2} a_{0}^{3}\right]$ & $Z$ & $\chi\left[\alpha^{2} a_{0}^{3}\right]$ \\
\hline 1 & $-5.999861546894(+0)$ & 47 & $-2.578631244428(-3)$ & 93 & $-5.589508155663(-4)$ \\
\hline 2 & $-1.499861548141(+0)$ & 48 & $-2.466676723115(-3)$ & 94 & $-5.443509574534(-4)$ \\
\hline 3 & $-6.665282168857(-1)$ & 49 & $-2.361509657648(-3)$ & 95 & $-5.302132488766(-4)$ \\
\hline 4 & $-3.748615531285(-1)$ & 50 & $-2.262592588983(-3)$ & 96 & $-5.165185776675(-4)$ \\
\hline 5 & $-2.398615568695(-1)$ & 51 & $-2.169440228389(-3)$ & 97 & $-5.032488119440(-4)$ \\
\hline 6 & $-1.665282281087(-1)$ & 52 & $-2.081613496469(-3)$ & 98 & $-4.903867404056(-4)$ \\
\hline 7 & $-1.223105464382(-1)$ & 53 & $-1.998714341935(-3)$ & 99 & $-4.779160168287(-4)$ \\
\hline 8 & $-9.361157308251(-2)$ & 54 & $-1.920381225688(-3)$ & 100 & $-4.658211084276(-4)$ \\
\hline 9 & $-7.393565422485(-2)$ & 55 & $-1.846285174354(-3)$ & 101 & $-4.540872477769(-4)$ \\
\hline 10 & $-5.986158805136(-2)$ & 56 & $-1.776126322645(-3)$ & 102 & $-4.427003880170(-4)$ \\
\hline 11 & $-4.944837364403(-2)$ & 57 & $-1.709630876565(-3)$ & 103 & $-4.316471610890(-4)$ \\
\hline 12 & $-4.152827301722(-2)$ & 58 & $-1.646548439917(-3)$ & 104 & $-4.209148387674(-4)$ \\
\hline 13 & $-3.536457532962(-2)$ & 59 & $-1.586649655271(-3)$ & 105 & $-4.104912962780(-4)$ \\
\hline 14 & $-3.047387288039(-2)$ & 60 & $-1.529724117834(-3)$ & 106 & $-4.003649783072(-4)$ \\
\hline 15 & $-2.652830671566(-2)$ & 61 & $-1.475578526709(-3)$ & 107 & $-3.905248672245(-4)$ \\
\hline 16 & $-2.329915294981(-2)$ & 62 & $-1.424035043188(-3)$ & 108 & $-3.809604533555(-4)$ \\
\hline 17 & $-2.062291236005(-2)$ & 63 & $-1.374929829983(-3)$ & 109 & $-3.716617071546(-4)$ \\
\hline 18 & $-1.838019977443(-2)$ & 64 & $-1.328111748960(-3)$ & 110 & $-3.626190531412(-4)$ \\
\hline 19 & $-1.648219527707(-2)$ & 65 & $-1.283441198032(-3)$ & 111 & $-3.538233454717(-4)$ \\
\hline 20 & $-1.486171290440(-2)$ & 66 & $-1.240789070458(-3)$ & 112 & $-3.452658450320(-4)$ \\
\hline 21 & $-1.346717216017(-2)$ & 67 & $-1.200035822051(-3)$ & 113 & $-3.369381979421(-4)$ \\
\hline 22 & $-1.225844211425(-2)$ & 68 & $-1.161070633696(-3)$ & 114 & $-3.288324153757(-4)$ \\
\hline 23 & $-1.120392166266(-2)$ & 69 & $-1.123790658208(-3)$ & 115 & $-3.209408546024(-4)$ \\
\hline 24 & $-1.027845291207(-2)$ & 70 & $-1.088100341950(-3)$ & 116 & $-3.132562011690(-4)$ \\
\hline 25 & $-9.461806676594(-3)$ & 71 & $-1.053910812872(-3)$ & 117 & $-3.057714521429(-4)$ \\
\hline 26 & $-8.737567592428(-3)$ & 72 & $-1.021139327613(-3)$ & 118 & $-2.984799003449(-4)$ \\
\hline 27 & $-8.092302733582(-3)$ & 73 & $-9.897087712636(-4)$ & 119 & $-2.913751195060(-4)$ \\
\hline 28 & $-7.514934235489(-3)$ & 74 & $-9.595472041212(-4)$ & 120 & $-2.844509502864(-4)$ \\
\hline 29 & $-6.996260657708(-3)$ & 75 & $-9.305874504615(-4)$ & 121 & $-2.777014871002(-4)$ \\
\hline 30 & $-6.528588108588(-3)$ & 76 & $-9.027667249364(-4)$ & 122 & $-2.711210656928(-4)$ \\
\hline 31 & $-6.105443280080(-3)$ & 77 & $-8.760262927126(-4)$ & 123 & $-2.647042514230(-4)$ \\
\hline 32 & $-5.721348246583(-3)$ & 78 & $-8.503111599116(-4)$ & 124 & $-2.584458282039(-4)$ \\
\hline 33 & $-5.371642289456(-3)$ & 79 & $-8.255697913049(-4)$ & 125 & $-2.523407880602(-4)$ \\
\hline 34 & $-5.052339850487(-3)$ & 80 & $-8.017538525544(-4)$ & 126 & $-2.463843212643(-4)$ \\
\hline 35 & $-4.760016478053(-3)$ & 81 & $-7.788179745891(-4)$ & 127 & $-2.405718070138(-4)$ \\
\hline 36 & $-4.491716634495(-3)$ & 82 & $-7.567195379726(-4)$ & 128 & $-2.348988046166(-4)$ \\
\hline 37 & $-4.244878703764(-3)$ & 83 & $-7.354184753456(-4)$ & 129 & $-2.293610451532(-4)$ \\
\hline 38 & $-4.017273627275(-3)$ & 84 & $-7.148770902326(-4)$ & 130 & $-2.239544235860(-4)$ \\
\hline 39 & $-3.806954409320(-3)$ & 85 & $-6.950598906817(-4)$ & 131 & $-2.186749912890(-4)$ \\
\hline 40 & $-3.612214346049(-3)$ & 86 & $-6.759334363662(-4)$ & 132 & $-2.135189489721(-4)$ \\
\hline 41 & $-3.431552297178(-3)$ & 87 & $-6.574661979166(-4)$ & 133 & $-2.084826399770(-4)$ \\
\hline 42 & $-3.263643675350(-3)$ & 88 & $-6.396284273783(-4)$ & 134 & $-2.035625439216(-4)$ \\
\hline 43 & $-3.107316102083(-3)$ & 89 & $-6.223920388001(-4)$ & 135 & $-1.987552706735(-4)$ \\
\hline 44 & $-2.961528891718(-3)$ & 90 & $-6.057304980587(-4)$ & 136 & $-1.940575546321(-4)$ \\
\hline 45 & $-2.825355690580(-3)$ & 91 & $-5.896187211102(-4)$ & 137 & $-1.894662493029(-4)$ \\
\hline 46 & $-2.697969728727(-3)$ & 92 & $-5.740329799424(-4)$ & & \\
\hline
\end{tabular}


Table 6

Relativistic magnetizabilities $\chi$ (in the units of $\alpha^{2} a_{0}^{3}$ ) of hydrogenlike atoms in the excited state $3 s_{1 / 2}$, obtained with $\alpha^{-1}=137.035999139$ (CODATA 2014).

\begin{tabular}{|c|c|c|c|c|c|}
\hline$Z$ & $\chi\left[\alpha^{2} a_{0}^{3}\right]$ & $Z$ & $\chi\left[\alpha^{2} a_{0}^{3}\right]$ & $Z$ & $\chi\left[\alpha^{2} a_{0}^{3}\right]$ \\
\hline 1 & $-3.449899704639(+1)$ & 47 & $-1.460589595746(-2)$ & 93 & $-2.938787161517(-3)$ \\
\hline 2 & $-8.623997035001(+0)$ & 48 & $-1.396150304355(-2)$ & 94 & $-2.852875528084(-3)$ \\
\hline 3 & $-3.832330349350(+0)$ & 49 & $-1.335612093153(-2)$ & 95 & $-2.769568453095(-3)$ \\
\hline 4 & $-2.155246989428(+0)$ & 50 & $-1.278665898809(-2)$ & 96 & $-2.688753878288(-3)$ \\
\hline 5 & $-1.378996955223(+0)$ & 51 & $-1.225032654958(-2)$ & 97 & $-2.610325233432(-3)$ \\
\hline 6 & $-9.573302467217(-1)$ & 52 & $-1.174459864603(-2)$ & 98 & $-2.534181079133(-3)$ \\
\hline 7 & $-7.030784965588(-1)$ & 53 & $-1.126718620861(-2)$ & 99 & $-2.460224772109(-3)$ \\
\hline 8 & $-5.380593067547(-1)$ & 54 & $-1.081601010268(-2)$ & 100 & $-2.388364150764(-3)$ \\
\hline 9 & $-4.249226678373(-1)$ & 55 & $-1.038917843515(-2)$ & 101 & $-2.318511239016(-3)$ \\
\hline 10 & $-3.439966693483(-1)$ & 56 & $-9.984966672575(-3)$ & 102 & $-2.250581966414(-3)$ \\
\hline 11 & $-2.841205559773(-1)$ & 57 & $-9.601800179014(-3)$ & 103 & $-2.184495902683(-3)$ \\
\hline 12 & $-2.385798342713(-1)$ & 58 & $-9.238238842908(-3)$ & 104 & $-2.120176004857(-3)$ \\
\hline 13 & $-2.031384168537(-1)$ & 59 & $-8.892963512056(-3)$ & 105 & $-2.057548375164(-3)$ \\
\hline 14 & $-1.750167094022(-1)$ & 60 & $-8.564763997698(-3)$ & 106 & $-1.996542027847(-3)$ \\
\hline 15 & $-1.523295228857(-1)$ & 61 & $-8.252528443623(-3)$ & 107 & $-1.937088662980(-3)$ \\
\hline 16 & $-1.337616949121(-1)$ & 62 & $-7.955233885600(-3)$ & 108 & $-1.879122445283(-3)$ \\
\hline 17 & $-1.183731048967(-1)$ & 63 & $-7.671937851189(-3)$ & 109 & $-1.822579785735(-3)$ \\
\hline 18 & $-1.054772880436(-1)$ & 64 & $-7.401770870923(-3)$ & 110 & $-1.767399123538(-3)$ \\
\hline 19 & $-9.456352977531(-2)$ & 65 & $-7.143929789539(-3)$ & 111 & $-1.713520705675(-3)$ \\
\hline 20 & $-8.524551073618(-2)$ & 66 & $-6.897671781034(-3)$ & 112 & $-1.660886360777(-3)$ \\
\hline 21 & $-7.722664300383(-2)$ & 67 & $-6.662308984122(-3)$ & 113 & $-1.609439263445(-3)$ \\
\hline 22 & $-7.027617365691(-2)$ & 68 & $-6.437203685646(-3)$ & 114 & $-1.559123684256(-3)$ \\
\hline 23 & $-6.421239627038(-2)$ & 69 & $-6.221763988858(-3)$ & 115 & $-1.509884719554(-3)$ \\
\hline 24 & $-5.889065287630(-2)$ & 70 & $-6.015439911547(-3)$ & 116 & $-1.461667993506(-3)$ \\
\hline 25 & $-5.419462557019(-2)$ & 71 & $-5.817719865910(-3)$ & 117 & $-1.414419322728(-3)$ \\
\hline 26 & $-5.002992592011(-2)$ & 72 & $-5.628127478035(-3)$ & 118 & $-1.368084330712(-3)$ \\
\hline 27 & $-4.631931450713(-2)$ & 73 & $-5.446218710006(-3)$ & 119 & $-1.322607994990(-3)$ \\
\hline 28 & $-4.299909351091(-2)$ & 74 & $-5.271579252139(-3)$ & 120 & $-1.277934103803(-3)$ \\
\hline 29 & $-4.001635454512(-2)$ & 75 & $-5.103822156684(-3)$ & 121 & $-1.234004590068(-3)$ \\
\hline 30 & $-3.732685762416(-2)$ & 76 & $-4.942585687741(-3)$ & 122 & $-1.190758697155(-3)$ \\
\hline 31 & $-3.489338111317(-2)$ & 77 & $-4.787531365038(-3)$ & 123 & $-1.148131910811(-3)$ \\
\hline 32 & $-3.268442682131(-2)$ & 78 & $-4.638342181782(-3)$ & 124 & $-1.106054560335(-3)$ \\
\hline 33 & $-3.067319549254(-2)$ & 79 & $-4.494720979055(-3)$ & 125 & $-1.064449942217(-3)$ \\
\hline 34 & $-2.883677003789(-2)$ & 80 & $-4.356388961144(-3)$ & 126 & $-1.023231737424(-3)$ \\
\hline 35 & $-2.715545972578(-2)$ & 81 & $-4.223084337950(-3)$ & 127 & $-9.823003535377(-4)$ \\
\hline 36 & $-2.561227007420(-2)$ & 82 & $-4.094561082102(-3)$ & 128 & $-9.415375740697(-4)$ \\
\hline 37 & $-2.419247164455(-2)$ & 83 & $-3.970587789747(-3)$ & 129 & $-9.007984322462(-4)$ \\
\hline 38 & $-2.288324719758(-2)$ & 84 & $-3.850946635148(-3)$ & 130 & $-8.598983050522(-4)$ \\
\hline 39 & $-2.167340134934(-2)$ & 85 & $-3.735432410250(-3)$ & 131 & $-8.185912607096(-4)$ \\
\hline 40 & $-2.055312038761(-2)$ & 86 & $-3.623851641304(-3)$ & 132 & $-7.765311175512(-4)$ \\
\hline 41 & $-1.951377258400(-2)$ & 87 & $-3.516021775421(-3)$ & 133 & $-7.331946777324(-4)$ \\
\hline 42 & $-1.854774138255(-2)$ & 88 & $-3.411770430663(-3)$ & 134 & $-6.877096795936(-4)$ \\
\hline 43 & $-1.764828542120(-2)$ & 89 & $-3.310934703898(-3)$ & 135 & $-6.383858465176(-4)$ \\
\hline 44 & $-1.680942056420(-2)$ & 90 & $-3.213360531211(-3)$ & 136 & $-5.808962689597(-4)$ \\
\hline 45 & $-1.602582007708(-2)$ & 91 & $-3.118902096172(-3)$ & 137 & $-4.831496808028(-4)$ \\
\hline 46 & $-1.529272982383(-2)$ & 92 & $-3.027421281680(-3)$ & & \\
\hline
\end{tabular}


Table 7

Relativistic magnetizabilities $\chi$ (in the units of $\alpha^{2} a_{0}^{3}$ ) of hydrogenlike atoms in the excited state $3 p_{1 / 2}$, obtained with $\alpha^{-1}=137.035999139$ (CODATA 2014).

\begin{tabular}{|c|c|c|c|c|c|}
\hline$Z$ & $\chi\left[\alpha^{2} a_{0}^{3}\right]$ & $Z$ & $\chi\left[\alpha^{2} a_{0}^{3}\right]$ & $Z$ & $\chi\left[\alpha^{2} a_{0}^{3}\right]$ \\
\hline 1 & $2.253079648737(+5)$ & 47 & $2.961501640474(-2)$ & 93 & $-5.821665076994(-4)$ \\
\hline 2 & $1.407454544846(+4)$ & 48 & $2.660256095643(-2)$ & 94 & $-6.134954630563(-4)$ \\
\hline 3 & $2.777786384569(+3)$ & 49 & $2.391571609084(-2)$ & 95 & $-6.412175427710(-4)$ \\
\hline 4 & $8.778590781585(+2)$ & 50 & $2.151495639188(-2)$ & 96 & $-6.656114912087(-4)$ \\
\hline 5 & $3.590183626425(+2)$ & 51 & $1.936614686446(-2)$ & 97 & $-6.869336974364(-4)$ \\
\hline 6 & $1.728120707372(+2)$ & 52 & $1.743972583622(-2)$ & 98 & $-7.054201043586(-4)$ \\
\hline 7 & $9.307202556408(+1)$ & 53 & $1.571002397351(-2)$ & 99 & $-7.212879385637(-4)$ \\
\hline 8 & $5.441676605826(+1)$ & 54 & $1.415469474468(-2)$ & 100 & $-7.347372789149(-4)$ \\
\hline 9 & $3.387288715058(+1)$ & 55 & $1.275423648472(-2)$ & 101 & $-7.459524799211(-4)$ \\
\hline 10 & $2.215129173089(+1)$ & 56 & $1.149159002773(-2)$ & 102 & $-7.551034641605(-4)$ \\
\hline 11 & $1.507478827498(+1)$ & 57 & $1.035179890189(-2)$ & 103 & $-7.623468964634(-4)$ \\
\hline 12 & $1.060143677914(+1)$ & 58 & $9.321721496853(-3)$ & 104 & $-7.678272511731(-4)$ \\
\hline 13 & $7.663506103714(+0)$ & 59 & $8.389786548922(-3)$ & 105 & $-7.716777825625(-4)$ \\
\hline 14 & $5.670768326413(+0)$ & 60 & $7.545784844853(-3)$ & 106 & $-7.740214073819(-4)$ \\
\hline 15 & $4.281366400764(+0)$ & 61 & $6.780691301789(-3)$ & 107 & $-7.749715075145(-4)$ \\
\hline 16 & $3.289268464602(+0)$ & 62 & $6.086512598482(-3)$ & 108 & $-7.746326598248(-4)$ \\
\hline 17 & $2.565971603606(+0)$ & 63 & $5.456156360839(-3)$ & 109 & $-7.731012994635(-4)$ \\
\hline 18 & $2.028897544840(+0)$ & 64 & $4.883318580250(-3)$ & 110 & $-7.704663221488(-4)$ \\
\hline 19 & $1.623567553290(+0)$ & 65 & $4.362386496197(-3)$ & 111 & $-7.668096302449(-4)$ \\
\hline 20 & $1.313194222044(+0)$ & 66 & $3.888354628892(-3)$ & 112 & $-7.622066267991(-4)$ \\
\hline 21 & $1.072414887772(+0)$ & 67 & $3.456752021987(-3)$ & 113 & $-7.567266610665(-4)$ \\
\hline 22 & $8.834142382734(-1)$ & 68 & $3.063579064713(-3)$ & 114 & $-7.504334284149(-4)$ \\
\hline 23 & $7.334657878250(-1)$ & 69 & $2.705252519197(-3)$ & 115 & $-7.433853268585(-4)$ \\
\hline 24 & $6.133382373914(-1)$ & 70 & $2.378557591857(-3)$ & 116 & $-7.356357717702(-4)$ \\
\hline 25 & $5.162415989039(-1)$ & 71 & $2.080606065368(-3)$ & 117 & $-7.272334695573(-4)$ \\
\hline 26 & $4.371174602330(-1)$ & 72 & $1.808799656204(-3)$ & 118 & $-7.182226501780(-4)$ \\
\hline 27 & $3.721529868818(-1)$ & 73 & $1.560797887120(-3)$ & 119 & $-7.086432572860(-4)$ \\
\hline 28 & $3.184430030589(-1)$ & 74 & $1.334489868466(-3)$ & 120 & $-6.985310933961(-4)$ \\
\hline 29 & $2.737517008702(-1)$ & 75 & $1.127969470206(-3)$ & 121 & $-6.879179156326(-4)$ \\
\hline 30 & $2.363424023942(-1)$ & 76 & $9.395134407774(-4)$ & 122 & $-6.768314751256(-4)$ \\
\hline 31 & $2.048544626550(-1)$ & 77 & $7.675620917952(-4)$ & 123 & $-6.652954896115(-4)$ \\
\hline 32 & $1.782132551088(-1)$ & 78 & $6.107022208586(-4)$ & 124 & $-6.533295337156(-4)$ \\
\hline 33 & $1.555636565334(-1)$ & 79 & $4.676519900050(-4)$ & 125 & $-6.409488238433(-4)$ \\
\hline 34 & $1.362204137883(-1)$ & 80 & $3.372475159160(-4)$ & 126 & $-6.281638630345(-4)$ \\
\hline 35 & $1.196307672315(-1)$ & 81 & $2.184309608788(-4)$ & 127 & $-6.149798928825(-4)$ \\
\hline 36 & $1.053460613117(-1)$ & 82 & $1.102399416397(-4)$ & 128 & $-6.013960698459(-4)$ \\
\hline 37 & $9.300000655504(-2)$ & 83 & $1.179809739097(-5)$ & 129 & $-5.874042329500(-4)$ \\
\hline 38 & $8.229190749879(-2)$ & 84 & $-7.769332117906(-5)$ & 130 & $-5.729870412697(-4)$ \\
\hline 39 & $7.297362892179(-2)$ & 85 & $-1.589629620510(-4)$ & 131 & $-5.581150963677(-4)$ \\
\hline 40 & $6.483939821434(-2)$ & 86 & $-2.326760862022(-4)$ & 132 & $-5.427423489936(-4)$ \\
\hline 41 & $5.771777535698(-2)$ & 87 & $-2.994405778162(-4)$ & 133 & $-5.267984479616(-4)$ \\
\hline 42 & $5.146529116126(-2)$ & 88 & $-3.598123306543(-4)$ & 134 & $-5.101753606939(-4)$ \\
\hline 43 & $4.596137798548(-2)$ & 89 & $-4.143000809618(-4)$ & 135 & $-4.927033391057(-4)$ \\
\hline 44 & $4.110430810152(-2)$ & 90 & $-4.633697486914(-4)$ & 136 & $-4.741197416352(-4)$ \\
\hline 45 & $3.680792236720(-2)$ & 91 & $-5.074483413545(-4)$ & 137 & $-4.553361480661(-4)$ \\
\hline 46 & $3.299898227783(-2)$ & 92 & $-5.469274683186(-4)$ & & \\
\hline
\end{tabular}


Table 8

Relativistic magnetizabilities $\chi$ (in the units of $\left.\alpha^{2} a_{0}^{3}\right)$ of hydrogenlike atoms in the excited state $3 p_{3 / 2}(\mu= \pm 1 / 2)$, obtained with $\alpha^{-1}=$ 137.035999139 (CODATA 2014).

\begin{tabular}{|c|c|c|c|c|c|}
\hline$Z$ & $\chi\left[\alpha^{2} a_{0}^{3}\right]$ & $Z$ & $\chi\left[\alpha^{2} a_{0}^{3}\right]$ & $Z$ & $\chi\left[\alpha^{2} a_{0}^{3}\right]$ \\
\hline 1 & $-2.253619636543(+5)$ & 47 & $-5.283002601438(-2)$ & 93 & $-4.382984551435(-3)$ \\
\hline 2 & $-1.408804422899(+4)$ & 48 & $-4.880910212206(-2)$ & 94 & $-4.217586852505(-3)$ \\
\hline 3 & $-2.783785165072(+3)$ & 49 & $-4.517485297588(-2)$ & 95 & $-4.059881012262(-3)$ \\
\hline 4 & $-8.812328586296(+2)$ & 50 & $-4.188291569502(-2)$ & 96 & $-3.909413039128(-3)$ \\
\hline 5 & $-3.611771430726(+2)$ & 51 & $-3.889478733899(-2)$ & 97 & $-3.765759884777(-3)$ \\
\hline 6 & $-1.743108511171(+2)$ & 52 & $-3.617695415994(-2)$ & 98 & $-3.628526974695(-3)$ \\
\hline 7 & $-9.417284670092(+1)$ & 53 & $-3.370016398695(-2)$ & 99 & $-3.497345952933(-3)$ \\
\hline 8 & $-5.525929631019(+1)$ & 54 & $-3.143881604543(-2)$ & 100 & $-3.371872619588(-3)$ \\
\hline 9 & $-3.453833399136(+1)$ & 55 & $-2.937044750287(-2)$ & 101 & $-3.251785041677(-3)$ \\
\hline 10 & $-2.269007181791(+1)$ & 56 & $-2.747529998186(-2)$ & 102 & $-3.136781820031(-3)$ \\
\hline 11 & $-1.551984925732(+1)$ & 57 & $-2.573595242306(-2)$ & 103 & $-3.026580496432(-3)$ \\
\hline 12 & $-1.097521666397(+1)$ & 58 & $-2.413700919040(-2)$ & 104 & $-2.920916086707(-3)$ \\
\hline 13 & $-7.981812500582(+0)$ & 59 & $-2.266483432400(-2)$ & 105 & $-2.819539726672(-3)$ \\
\hline 14 & $-5.945058175495(+0)$ & 60 & $-2.130732446792(-2)$ & 106 & $-2.722217418896(-3)$ \\
\hline 15 & $-4.520145911594(+0)$ & 61 & $-2.005371431041(-2)$ & 107 & $-2.628728 \quad 869054(-3)$ \\
\hline 16 & $-3.498985331674(+0)$ & 62 & $-1.889440943867(-2)$ & 108 & $-2.538866401341(-3)$ \\
\hline 17 & $-2.751602028338(+0)$ & 63 & $-1.782084237633(-2)$ & 109 & $-2.452433942839(-3)$ \\
\hline 18 & $-2.194343262026(+0)$ & 64 & $-1.682534828021(-2)$ & 110 & $-2.369246067020(-3)$ \\
\hline 19 & $-1.771930918400(+0)$ & 65 & $-1.590105735359(-2)$ & 111 & $-2.289127086553(-3)$ \\
\hline 20 & $-1.446972916787(+0)$ & 66 & $-1.504180151118(-2)$ & 112 & $-2.211910185318(-3)$ \\
\hline 21 & $-1.193642369298(+0)$ & 67 & $-1.424203322520(-2)$ & 113 & $-2.137436578922(-3)$ \\
\hline 22 & $-9.937627852646(-1)$ & 68 & $-1.349675480860(-2)$ & 114 & $-2.065554691917(-3)$ \\
\hline 23 & $-8.343232689235(-1)$ & 69 & $-1.280145666232(-2)$ & 115 & $-1.996119338262(-3)$ \\
\hline 24 & $-7.058661000903(-1)$ & 70 & $-1.215206323948(-2)$ & 116 & $-1.928990889064(-3)$ \\
\hline 25 & $-6.014192279083(-1)$ & 71 & $-1.154488566765(-2)$ & 117 & $-1.864034408017(-3)$ \\
\hline 26 & $-5.157765018528(-1)$ & 72 & $-1.097658012822(-2)$ & 118 & $-1.801118729662(-3)$ \\
\hline 27 & $-4.450041909658(-1)$ & 73 & $-1.044411122443(-2)$ & 119 & $-1.740115447942(-3)$ \\
\hline 28 & $-3.860974185415(-1)$ & 74 & $-9.944719681017(-3)$ & 120 & $-1.680897771276(-3)$ \\
\hline 29 & $-3.367375635824(-1)$ & 75 & $-9.475893812523(-3)$ & 121 & $-1.623339183709(-3)$ \\
\hline 30 & $-2.951187029957(-1)$ & 76 & $-9.035344276842(-3)$ & 122 & $-1.567311826495(-3)$ \\
\hline 31 & $-2.598219318800(-1)$ & 77 & $-8.620981698059(-3)$ & 123 & $-1.512684475701(-3)$ \\
\hline 32 & $-2.297233222014(-1)$ & 78 & $-8.230896799857(-3)$ & 124 & $-1.459319930243(-3)$ \\
\hline 33 & $-2.039258041026(-1)$ & 79 & $-7.863342739572(-3)$ & 125 & $-1.407071525691(-3)$ \\
\hline 34 & $-1.817082542449(-1)$ & 80 & $-7.516719374597(-3)$ & 126 & $-1.355778323459(-3)$ \\
\hline 35 & $-1.624870927398(-1)$ & 81 & $-7.189559228432(-3)$ & 127 & $-1.305258237710(-3)$ \\
\hline 36 & $-1.457870640073(-1)$ & 82 & $-6.880514954158(-3)$ & 128 & $-1.255297842467(-3)$ \\
\hline 37 & $-1.312188238309(-1)$ & 83 & $-6.588348119300(-3)$ & 129 & $-1.205636612851(-3)$ \\
\hline 38 & $-1.184616150147(-1)$ & 84 & $-6.311919158563(-3)$ & 130 & $-1.155941357871(-3)$ \\
\hline 39 & $-1.072497791613(-1)$ & 85 & $-6.050178360340(-3)$ & 131 & $-1.105762264352(-3)$ \\
\hline 40 & $-9.736218310255(-2)$ & 86 & $-5.802157769625(-3)$ & 132 & $-1.054451640406(-3)$ \\
\hline 41 & $-8.861387632359(-2)$ & 87 & $-5.566963904484(-3)$ & 133 & $-1.000998727871(-3)$ \\
\hline 42 & $-8.084946810726(-2)$ & 88 & $-5.343771195769(-3)$ & 134 & $-9.436464304010(-4)$ \\
\hline 43 & $-7.393783915312(-2)$ & 89 & $-5.131816070673(-3)$ & 135 & $-8.788040064270(-4)$ \\
\hline 44 & $-6.776789529949(-2)$ & 90 & $-4.930391610179(-3)$ & 136 & $-7.966180491771(-4)$ \\
\hline 45 & $-6.224513994779(-2)$ & 91 & $-4.738842718716(-3)$ & 137 & $-6.139274383931(-4)$ \\
\hline 46 & $-5.728889337946(-2)$ & 92 & $-4.556561751516(-3)$ & & \\
\hline
\end{tabular}


Table 9

Relativistic magnetizabilities $\chi$ (in the units of $\alpha^{2} a_{0}^{3}$ ) of hydrogenlike atoms in the excited state $3 p_{3 / 2}(\mu= \pm 3 / 2)$, obtained with $\alpha^{-1}=$ 137.035999139 (CODATA 2014).

\begin{tabular}{|c|c|c|c|c|c|}
\hline$Z$ & $\chi\left[\alpha^{2} a_{0}^{3}\right]$ & $Z$ & $\chi\left[\alpha^{2} a_{0}^{3}\right]$ & $Z$ & $\chi\left[\alpha^{2} a_{0}^{3}\right]$ \\
\hline 1 & $-3.599951541316(+1)$ & 47 & $-1.581344317096(-2)$ & 93 & $-3.681907779187(-3)$ \\
\hline 2 & $-8.999515414600(+0)$ & 48 & $-1.514152196291(-2)$ & 94 & $-3.593908592141(-3)$ \\
\hline 3 & $-3.999515417007(+0)$ & 49 & $-1.451032127476(-2)$ & 95 & $-3.508677457715(-3)$ \\
\hline 4 & $-2.249515420376(+0)$ & 50 & $-1.391661634388(-2)$ & 96 & $-3.426099682411(-3)$ \\
\hline 5 & $-1.439515424709(+0)$ & 51 & $-1.335749542934(-2)$ & 97 & $-3.346066454083(-3)$ \\
\hline 6 & $-9.995154300037(-1)$ & 52 & $-1.283032404604(-2)$ & 98 & $-3.268474483707(-3)$ \\
\hline 7 & $-7.342093138125(-1)$ & 53 & $-1.233271387727(-2)$ & 99 & $-3.193225672345(-3)$ \\
\hline 8 & $-5.620154434822(-1)$ & 54 & $-1.186249567930(-2)$ & 100 & $-3.120226801306(-3)$ \\
\hline 9 & $-4.439598961100(-1)$ & 55 & $-1.141769560251(-2)$ & 101 & $-3.049389243671(-3)$ \\
\hline 10 & $-3.595154608118(-1)$ & 56 & $-1.099651444560(-2)$ & 102 & $-2.980628695521(-3)$ \\
\hline 11 & $-2.970361320778(-1)$ & 57 & $-1.059730943482(-2)$ & 103 & $-2.913864925341(-3)$ \\
\hline 12 & $-2.495154819926(-1)$ & 58 & $-1.021857818298(-2)$ & 104 & $-2.849021540206(-3)$ \\
\hline 13 & $-2.125332455065(-1)$ & 59 & $-9.858944535296(-3)$ & 105 & $-2.786025767482(-3)$ \\
\hline 14 & $-1.831889764125(-1)$ & 60 & $-9.517146052552(-3)$ & 106 & $-2.724808250876(-3)$ \\
\hline 15 & $-1.595155209851(-1)$ & 61 & $-9.192022918728(-3)$ & 107 & $-2.665302859760(-3)$ \\
\hline 16 & $-1.401405359083(-1)$ & 62 & $-8.882508090695(-3)$ & 108 & $-2.607446510804(-3)$ \\
\hline 17 & $-1.240830258428(-1)$ & 63 & $-8.587618533612(-3)$ & 109 & $-2.551179001003(-3)$ \\
\hline 18 & $-1.106266797544(-1)$ & 64 & $-8.306447407350(-3)$ & 110 & $-2.496442851286(-3)$ \\
\hline 19 & $-9.923857814497(-2)$ & 65 & $-8.038157087817(-3)$ & 111 & $-2.443183159938(-3)$ \\
\hline 20 & $-8.951560523006(-2)$ & 66 & $-7.781972922763(-3)$ & 112 & $-2.391347465147(-3)$ \\
\hline 21 & $-8.114827802908(-2)$ & 67 & $-7.537177635034(-3)$ & 113 & $-2.340885616025(-3)$ \\
\hline 22 & $-7.389581095789(-2)$ & 68 & $-7.303106297671(-3)$ & 114 & $-2.291749651510(-3)$ \\
\hline 23 & $-6.756859738911(-2)$ & 69 & $-7.079141815033(-3)$ & 115 & $-2.243893686616(-3)$ \\
\hline 24 & $-6.201568995919(-2)$ & 70 & $-6.864710852549(-3)$ & 116 & $-2.197273805507(-3)$ \\
\hline 25 & $-5.711571354902(-2)$ & 71 & $-6.659280164896(-3)$ & 117 & $-2.151847960948(-3)$ \\
\hline 26 & $-5.277017597173(-2)$ & 72 & $-6.462353278650(-3)$ & 118 & $-2.107575879694(-3)$ \\
\hline 27 & $-4.889847966727(-2)$ & 73 & $-6.273467490837(-3)$ & 119 & $-2.064418973414(-3)$ \\
\hline 28 & $-4.543415744391(-2)$ & 74 & $-6.092191149461(-3)$ & 120 & $-2.022340254799(-3)$ \\
\hline 29 & $-4.232200065452(-2)$ & 75 & $-5.918121186140(-3)$ & 121 & $-1.981304258498(-3)$ \\
\hline 30 & $-3.951584594454(-2)$ & 76 & $-5.750880874483(-3)$ & 122 & $-1.941276966567(-3)$ \\
\hline 31 & $-3.697685346084(-2)$ & 77 & $-5.590117790918(-3)$ & 123 & $-1.902225738155(-3)$ \\
\hline 32 & $-3.467215564481(-2)$ & 78 & $-5.435501957331(-3)$ & 124 & $-1.864119243131(-3)$ \\
\hline 33 & $-3.257378817943(-2)$ & 79 & $-5.286724147230(-3)$ & 125 & $-1.826927399420(-3)$ \\
\hline 34 & $-3.065783771005(-2)$ & 80 & $-5.143494339187(-3)$ & 126 & $-1.790621313804(-3)$ \\
\hline 35 & $-2.890375752143(-2)$ & 81 & $-5.005540303093(-3)$ & 127 & $-1.755173225972(-3)$ \\
\hline 36 & $-2.729381438190(-2)$ & 82 & $-4.872606306358(-3)$ & 128 & $-1.720556455618(-3)$ \\
\hline 37 & $-2.581263858925(-2)$ & 83 & $-4.744451928563(-3)$ & 129 & $-1.686745352401(-3)$ \\
\hline 38 & $-2.444685578593(-2)$ & 84 & $-4.620850974296(-3)$ & 130 & $-1.653715248583(-3)$ \\
\hline 39 & $-2.318478399143(-2)$ & 85 & $-4.501590474984(-3)$ & 131 & $-1.621442414196(-3)$ \\
\hline 40 & $-2.201618297622(-2)$ & 86 & $-4.386469771504(-3)$ & 132 & $-1.589904014568(-3)$ \\
\hline 41 & $-2.093204589197(-2)$ & 87 & $-4.275299670162(-3)$ & 133 & $-1.559078070083(-3)$ \\
\hline 42 & $-1.992442520773(-2)$ & 88 & $-4.167901665443(-3)$ & 134 & $-1.528943418030(-3)$ \\
\hline 43 & $-1.898628664563(-2)$ & 89 & $-4.064107223530(-3)$ & 135 & $-1.499479676428(-3)$ \\
\hline 44 & $-1.811138608454(-2)$ & 90 & $-3.963757121251(-3)$ & 136 & $-1.470667209701(-3)$ \\
\hline 45 & $-1.729416539503(-2)$ & 91 & $-3.866700835581(-3)$ & 137 & $-1.442487096101(-3)$ \\
\hline 46 & $-1.652966394988(-2)$ & 92 & $-3.772795979350(-3)$ & & \\
\hline
\end{tabular}


Table 10

Relativistic magnetizabilities $\chi$ (in the units of $\left.\alpha^{2} a_{0}^{3}\right)$ of hydrogenlike atoms in the excited state $3 d_{3 / 2}(\mu= \pm 1 / 2)$, obtained with $\alpha^{-1}=$ 137.035999139 (CODATA 2014).

\begin{tabular}{|c|c|c|c|c|c|}
\hline$Z$ & $\chi\left[\alpha^{2} a_{0}^{3}\right]$ & $Z$ & $\chi\left[\alpha^{2} a_{0}^{3}\right]$ & $Z$ & $\chi\left[\alpha^{2} a_{0}^{3}\right]$ \\
\hline 1 & $7.300965637634(+5)$ & 47 & $1.382152566398(-1)$ & 93 & $7.014861885531(-3)$ \\
\hline 2 & $4.562621482550(+4)$ & 48 & $1.266106404598(-1)$ & 94 & $6.668966264598(-3)$ \\
\hline 3 & $9.010998791784(+3)$ & 49 & $1.161726868210(-1)$ & 95 & $6.342269233241(-3)$ \\
\hline 4 & $2.850433485628(+3)$ & 50 & $1.067644863689(-1)$ & 96 & $6.033549850614(-3)$ \\
\hline 5 & $1.167167467288(+3)$ & 51 & $9.826745342003(-2)$ & 97 & $5.741675544197(-3)$ \\
\hline 6 & $5.626520147653(+2)$ & 52 & $9.057858044552(-2)$ & 98 & $5.465594924698(-3)$ \\
\hline 7 & $3.035664451768(+2)$ & 53 & $8.360814639921(-2)$ & 99 & $5.204331249137(-3)$ \\
\hline 8 & $1.778510190602(+2)$ & 54 & $7.727779708599(-2)$ & 100 & $4.956976467939(-3)$ \\
\hline 9 & $1.109649855296(+2)$ & 55 & $7.151893169168(-2)$ & 101 & $4.722685798743(-3)$ \\
\hline 10 & $7.275535696625(+1)$ & 56 & $6.627134219887(-2)$ & 102 & $4.500672775791(-3)$ \\
\hline 11 & $4.965608395653(+1)$ & 57 & $6.148206243195(-2)$ & 103 & $4.290204729127(-3)$ \\
\hline 12 & $3.503205992112(+1)$ & 58 & $5.710439147319(-2)$ & 104 & $4.090598652646(-3)$ \\
\hline 13 & $2.541170970698(+1)$ & 59 & $5.309706260397(-2)$ & 105 & $3.901217424267(-3)$ \\
\hline 14 & $1.887473795509(+1)$ & 60 & $4.942353408648(-2)$ & 106 & $3.721466345294(-3)$ \\
\hline 15 & $1.430813127641(+1)$ & 61 & $4.605138227148(-2)$ & 107 & $3.550789969362(-3)$ \\
\hline 16 & $1.104058164226(+1)$ & 62 & $4.295178089985(-2)$ & 108 & $3.388669194380(-3)$ \\
\hline 17 & $8.653048412936(+0)$ & 63 & $4.009905321835(-2)$ & 109 & $3.234618593494(-3)$ \\
\hline 18 & $6.876012904102(+0)$ & 64 & $3.747028577856(-2)$ & 110 & $3.088183963528(-3)$ \\
\hline 19 & $5.531502038377(+0)$ & 65 & $3.504499463042(-2)$ & 111 & $2.948940071428(-3)$ \\
\hline 20 & $4.499218736289(+0)$ & 66 & $3.280483613677(-2)$ & 112 & $2.816488581157(-3)$ \\
\hline 21 & $3.696138953551(+0)$ & 67 & $3.073335588449(-2)$ & 113 & $2.690456145175(-3)$ \\
\hline 22 & $3.063880523391(+0)$ & 68 & $2.881577020174(-2)$ & 114 & $2.570492646153(-3)$ \\
\hline 23 & $2.560689477752(+0)$ & 69 & $2.703877564806(-2)$ & 115 & $2.456269575945(-3)$ \\
\hline 24 & $2.156242308687(+0)$ & 70 & $2.539038255788(-2)$ & 116 & $2.347478540039(-3)$ \\
\hline 25 & $1.828206482298(+0)$ & 71 & $2.385976931323(-2)$ & 117 & $2.243829876846(-3)$ \\
\hline 26 & $1.559922546230(+0)$ & 72 & $2.243715451954(-2)$ & 118 & $2.145051382123(-3)$ \\
\hline 27 & $1.338815810581(+0)$ & 73 & $2.111368467647(-2)$ & 119 & $2.050887129763(-3)$ \\
\hline 28 & $1.155291163955(+0)$ & 74 & $1.988133528657(-2)$ & 120 & $1.961096380949(-3)$ \\
\hline 29 & $1.001953132875(+0)$ & 75 & $1.873282364148(-2)$ & 121 & $1.875452574425(-3)$ \\
\hline 30 & $8.730482392226(-1)$ & 76 & $1.766153177485(-2)$ & 122 & $1.793742391259(-3)$ \\
\hline 31 & $7.640614424179(-1)$ & 77 & $1.666143828387(-2)$ & 123 & $1.715764888084(-3)$ \\
\hline 32 & $6.714207855885(-1)$ & 78 & $1.572705790080(-2)$ & 124 & $1.641330693342(-3)$ \\
\hline 33 & $5.922789538408(-1)$ & 79 & $1.485338784915(-2)$ & 125 & $1.570261261539(-3)$ \\
\hline 34 & $5.243501244773(-1)$ & 80 & $1.403586014978(-2)$ & 126 & $1.502388180971(-3)$ \\
\hline 35 & $4.657869895405(-1)$ & 81 & $1.327029915343(-2)$ & 127 & $1.437552530798(-3)$ \\
\hline 36 & $4.150872566405(-1)$ & 82 & $1.255288367207(-2)$ & 128 & $1.375604283784(-3)$ \\
\hline 37 & $3.710219834171(-1)$ & 83 & $1.188011316300(-2)$ & 129 & $1.316401751378(-3)$ \\
\hline 38 & $3.325802259926(-1)$ & 84 & $1.124877749015(-2)$ & 130 & $1.259811068366(-3)$ \\
\hline 39 & $2.989259784155(-1)$ & 85 & $1.065592984775(-2)$ & 131 & $1.205705714951(-3)$ \\
\hline 40 & $2.693644447883(-1)$ & 86 & $1.009886248361(-2)$ & 132 & $1.153966075355(-3)$ \\
\hline 41 & $2.433154503706(-1)$ & 87 & $9.575084904592(-3)$ & 133 & $1.104479034847(-3)$ \\
\hline 42 & $2.202923519639(-1)$ & 88 & $9.082304285923(-3)$ & 134 & $1.057137625440(-3)$ \\
\hline 43 & $1.998852127268(-1)$ & 89 & $8.618407839891(-3)$ & 135 & $1.011840763111(-3)$ \\
\hline 44 & $1.817473047893(-1)$ & 90 & $8.181446928965(-3)$ & 136 & $9.684933165501(-4)$ \\
\hline 45 & $1.655842243911(-1)$ & 91 & $7.769622733997(-3)$ & 137 & $9.270118604514(-4)$ \\
\hline 46 & $1.511450697722(-1)$ & 92 & $7.381273310506(-3)$ & & \\
\hline
\end{tabular}


Table 11

Relativistic magnetizabilities $\chi$ (in the units of $\left.\alpha^{2} a_{0}^{3}\right)$ of hydrogenlike atoms in the excited state $3 d_{3 / 2}(\mu= \pm 3 / 2)$, obtained with $\alpha^{-1}=$ 137.035999139 (CODATA 2014).

\begin{tabular}{|c|c|c|c|c|c|}
\hline$Z$ & $\chi\left[\alpha^{2} a_{0}^{3}\right]$ & $Z$ & $\chi\left[\alpha^{2} a_{0}^{3}\right]$ & $Z$ & $\chi\left[\alpha^{2} a_{0}^{3}\right]$ \\
\hline 1 & $4.867170427249(+5)$ & 47 & $8.602099797280(-2)$ & 93 & $3.270743993589(-3)$ \\
\hline 2 & $3.041397676633(+4)$ & 48 & $7.854587339729(-2)$ & 94 & $3.074334771839(-3)$ \\
\hline 3 & $6.005777188294(+3)$ & 49 & $7.183268773333(-2)$ & 95 & $2.889647729010(-3)$ \\
\hline 4 & $1.899414206410(+3)$ & 50 & $6.579142215135(-2)$ & 96 & $2.715913505579(-3)$ \\
\hline 5 & $7.775518608467(+2)$ & 51 & $6.034415187203(-2)$ & 97 & $2.552419366998(-3)$ \\
\hline 6 & $3.747126702718(+2)$ & 52 & $5.542322973071(-2)$ & 98 & $2.398504552941(-3)$ \\
\hline 7 & $2.020921318113(+2)$ & 53 & $5.096977048562(-2)$ & 99 & $2.253556048873(-3)$ \\
\hline 8 & $1.183488120139(+2)$ & 54 & $4.693238160067(-2)$ & 100 & $2.117004737932(-3)$ \\
\hline 9 & $7.380403348125(+1)$ & 55 & $4.326609680709(-2)$ & 101 & $1.988321895654(-3)$ \\
\hline 10 & $4.836378727173(+1)$ & 56 & $3.993147711516(-2)$ & 102 & $1.867015994085(-3)$ \\
\hline 11 & $3.298856944505(+1)$ & 57 & $3.689385059673(-2)$ & 103 & $1.752629785362(-3)$ \\
\hline 12 & $2.325770033699(+1)$ & 58 & $3.412266756745(-2)$ & 104 & $1.644737638004(-3)$ \\
\hline 13 & $1.685851550415(+1)$ & 59 & $3.159095205196(-2)$ & 105 & $1.542943101917(-3)$ \\
\hline 14 & $1.251194599180(+1)$ & 60 & $2.927483383938(-2)$ & 106 & $1.446876680612(-3)$ \\
\hline 15 & $9.476747878165(+0)$ & 61 & $2.715314820217(-2)$ & 107 & $1.356193791312(-3)$ \\
\hline 16 & $7.305916166512(+0)$ & 62 & $2.520709259449(-2)$ & 108 & $1.270572895610(-3)$ \\
\hline 17 & $5.720471928567(+0)$ & 63 & $2.341993147119(-2)$ & 109 & $1.189713785035(-3)$ \\
\hline 18 & $4.541014606832(+0)$ & 64 & $2.177674185902(-2)$ & 110 & $1.113336007491(-3)$ \\
\hline 19 & $3.649102729529(+0)$ & 65 & $2.026419353296(-2)$ & 111 & $1.041177421878(-3)$ \\
\hline 20 & $2.964695010925(+0)$ & 66 & $1.887035865415(-2)$ & 112 & $9.729928694670(-4)$ \\
\hline 21 & $2.432562442655(+0)$ & 67 & $1.758454655389(-2)$ & 113 & $9.085529516967(-4)$ \\
\hline 22 & $2.013877219041(+0)$ & 68 & $1.639716003255(-2)$ & 114 & $8.476429050609(-4)$ \\
\hline 23 & $1.680877097290(+0)$ & 69 & $1.529957011035(-2)$ & 115 & $7.900615646396(-4)$ \\
\hline 24 & $1.413405107189(+0)$ & 70 & $1.428400663920(-2)$ & 116 & $7.356204086288(-4)$ \\
\hline 25 & $1.196620094241(+0)$ & 71 & $1.334346257912(-2)$ & 117 & $6.841426769363(-4)$ \\
\hline 26 & $1.019454059393(+0)$ & 72 & $1.247161007222(-2)$ & 118 & $6.354625575598(-4)$ \\
\hline 27 & $8.735552195380(-1)$ & 73 & $1.166272672374(-2)$ & 119 & $5.894244350404(-4)$ \\
\hline 28 & $7.525526820974(-1)$ & 74 & $1.091163073215(-2)$ & 120 & $5.458821958062(-4)$ \\
\hline 29 & $6.515376014502(-1)$ & 75 & $1.021362370608(-2)$ & 121 & $5.046985856936(-4)$ \\
\hline 30 & $5.666922779316(-1)$ & 76 & $9.564440171373(-3)$ & 122 & $4.657446153561(-4)$ \\
\hline 31 & $4.950217888754(-1)$ & 77 & $8.960202911774(-3)$ & 123 & $4.288990096540(-4)$ \\
\hline 32 & $4.341576115282(-1)$ & 78 & $8.397383405591(-3)$ & 124 & $3.940476974653(-4)$ \\
\hline 33 & $3.822124109723(-1)$ & 79 & $7.872766721871(-3)$ & 125 & $3.610833386704(-4)$ \\
\hline 34 & $3.376716050471(-1)$ & 80 & $7.383420325875(-3)$ & 126 & $3.299048853472(-4)$ \\
\hline 35 & $2.993116455029(-1)$ & 81 & $6.926666317264(-3)$ & 127 & $3.004171744690(-4)$ \\
\hline 36 & $2.661379003360(-1)$ & 82 & $6.500056687449(-3)$ & 128 & $2.725305496322(-4)$ \\
\hline 37 & $2.373370517456(-1)$ & 83 & $6.101351236618(-3)$ & 129 & $2.461605095486(-4)$ \\
\hline 38 & $2.122403382851(-1)$ & 84 & $5.728497837415(-3)$ & 130 & $2.212273812330(-4)$ \\
\hline 39 & $1.902949656452(-1)$ & 85 & $5.379614772207(-3)$ & 131 & $1.976560159868(-4)$ \\
\hline 40 & $1.710417188720(-1)$ & 86 & $5.052974905373(-3)$ & 132 & $1.753755064402(-4)$ \\
\hline 41 & $1.540973174679(-1)$ & 87 & $4.746991481851(-3)$ & 133 & $1.543189230566(-4)$ \\
\hline 42 & $1.391404233396(-1)$ & 88 & $4.460205368939(-3)$ & 134 & $1.344230686367(-4)$ \\
\hline 43 & $1.259004808115(-1)$ & 89 & $4.191273580723(-3)$ & 135 & $1.156282494772(-4)$ \\
\hline 44 & $1.141487662390(-1)$ & 90 & $3.938958943899(-3)$ & 136 & $9.787806195005(-5)$ \\
\hline 45 & $1.036911719427(-1)$ & 91 & $3.702120780660(-3)$ & 137 & $8.111919336523(-5)$ \\
\hline 46 & $9.436235921464(-2)$ & 92 & $3.479706498989(-3)$ & & \\
\hline
\end{tabular}


Table 12

Relativistic magnetizabilities $\chi$ (in the units of $\left.\alpha^{2} a_{0}^{3}\right)$ of hydrogenlike atoms in the excited state $3 d_{5 / 2}(\mu= \pm 1 / 2)$, obtained with $\alpha^{-1}=$ 137.035999139 (CODATA 2014).

\begin{tabular}{|c|c|c|c|c|c|}
\hline$Z$ & $\chi\left[\alpha^{2} a_{0}^{3}\right]$ & $Z$ & $\chi\left[\alpha^{2} a_{0}^{3}\right]$ & $Z$ & $\chi\left[\alpha^{2} a_{0}^{3}\right]$ \\
\hline 1 & $-7.301295633993(+5)$ & 47 & $-1.527906889615(-1)$ & 93 & $-1.046863644964(-2)$ \\
\hline 2 & $-4.563446446141(+4)$ & 48 & $-1.405701300375(-1)$ & 94 & $-1.004204115620(-2)$ \\
\hline 3 & $-9.014665094365(+3)$ & 49 & $-1.295535604542(-1)$ & 95 & $-9.637181328664(-3)$ \\
\hline 4 & $-2.852495621544(+3)$ & 50 & $-1.196011148224(-1)$ & 96 & $-9.252730884592(-3)$ \\
\hline 5 & $-1.168487103207(+3)$ & 51 & $-1.105915383609(-1)$ & 97 & $-8.887457500952(-3)$ \\
\hline 6 & $-5.635683173539(+2)$ & 52 & $-1.024194085943(-1)$ & 98 & $-8.540215100647(-3)$ \\
\hline 7 & $-3.042395504902(+2)$ & 53 & $-9.499281518380(-2)$ & 99 & $-8.209937010306(-3)$ \\
\hline 8 & $-1.783662799902(+2)$ & 54 & $-8.823141545890(-2)$ & 100 & $-7.895629723348(-3)$ \\
\hline 9 & $-1.113720288720(+2)$ & 55 & $-8.206479924105(-2)$ & 101 & $-7.596367209358(-3)$ \\
\hline 10 & $-7.308499290675(+1)$ & 56 & $-7.643110924276(-2)$ & 102 & $-7.311285717086(-3)$ \\
\hline 11 & $-4.992844717585(+1)$ & 57 & $-7.127587341018(-2)$ & 103 & $-7.039579023927(-3)$ \\
\hline 12 & $-3.526086254104(+1)$ & 58 & $-6.655101363495(-2)$ & 104 & $-6.780494089633(-3)$ \\
\hline 13 & $-2.560661193968(+1)$ & 59 & $-6.221400172083(-2)$ & 105 & $-6.533327076369(-3)$ \\
\hline 14 & $-1.904274127036(+1)$ & 60 & $-5.822713869159(-2)$ & 106 & $-6.297419701108(-3)$ \\
\hline 15 & $-1.445443391981(+1)$ & 61 & $-5.455693773077(-2)$ & 107 & $-6.072155889783(-3)$ \\
\hline 16 & $-1.116912387797(+1)$ & 62 & $-5.117359445379(-2)$ & 108 & $-5.856958705706(-3)$ \\
\hline 17 & $-8.766871259421(+0)$ & 63 & $-4.805053098946(-2)$ & 109 & $-5.651287527467(-3)$ \\
\hline 18 & $-6.977500761360(+0)$ & 64 & $-4.516400261652(-2)$ & 110 & $-5.454635453992(-3)$ \\
\hline 19 & $-5.622550796875(+0)$ & 65 & $-4.249275756005(-2)$ & 111 & $-5.266526916613(-3)$ \\
\hline 20 & $-4.581354763687(+0)$ & 66 & $-4.001774208212(-2)$ & 112 & $-5.086515479939(-3)$ \\
\hline 21 & $-3.770604924779(+0)$ & 67 & $-3.772184426261(-2)$ & 113 & $-4.914181815081(-3)$ \\
\hline 22 & $-3.131698393258(+0)$ & 68 & $-3.558967091031(-2)$ & 114 & $-4.749131830333(-3)$ \\
\hline 23 & $-2.622707394993(+0)$ & 69 & $-3.360735291068(-2)$ & 115 & $-4.590994945836(-3)$ \\
\hline 24 & $-2.213170053618(+0)$ & 70 & $-3.176237503831(-2)$ & 116 & $-4.439422499982(-3)$ \\
\hline 25 & $-1.880642574707(+0)$ & 71 & $-3.004342686379(-2)$ & 117 & $-4.294086276479(-3)$ \\
\hline 26 & $-1.608375221403(+0)$ & 72 & $-2.844027188862(-2)$ & 118 & $-4.154677141989(-3)$ \\
\hline 27 & $-1.383719422704(+0)$ & 73 & $-2.694363246467(-2)$ & 119 & $-4.020903785201(-3)$ \\
\hline 28 & $-1.197019138954(+0)$ & 74 & $-2.554508841005(-2)$ & 120 & $-3.892491548994(-3)$ \\
\hline 29 & $-1.040828288750(+0)$ & 75 & $-2.423698753349(-2)$ & 121 & $-3.769181348118(-3)$ \\
\hline 30 & $-9.093510775626(-1)$ & 76 & $-2.301236653250(-2)$ & 122 & $-3.650728665479(-3)$ \\
\hline 31 & $-7.980368616128(-1)$ & 77 & $-2.186488094560(-2)$ & 123 & $-3.536902620742(-3)$ \\
\hline 32 & $-7.032835554317(-1)$ & 78 & $-2.078874302131(-2)$ & 124 & $-3.427485105480(-3)$ \\
\hline 33 & $-6.222182101673(-1)$ & 79 & $-1.977866652164(-2)$ & 125 & $-3.322269979655(-3)$ \\
\hline 34 & $-5.525330825458(-1)$ & 80 & $-1.882981761046(-2)$ & 126 & $-3.221062324594(-3)$ \\
\hline 35 & $-4.923620301194(-1)$ & 81 & $-1.793777109019(-2)$ & 127 & $-3.123677748114(-3)$ \\
\hline 36 & $-4.401865050347(-1)$ & 82 & $-1.709847134706(-2)$ & 128 & $-3.029941737748(-3)$ \\
\hline 37 & $-3.947634760365(-1)$ & 83 & $-1.630819744870(-2)$ & 129 & $-2.939689058414(-3)$ \\
\hline 38 & $-3.550697394179(-1)$ & 84 & $-1.556353190887(-2)$ & 130 & $-2.852763191148(-3)$ \\
\hline 39 & $-3.202585807440(-1)$ & 85 & $-1.486133269611(-2)$ & 131 & $-2.769015809816(-3)$ \\
\hline 40 & $-2.896258172386(-1)$ & 86 & $-1.419870811609(-2)$ & 132 & $-2.688306292963(-3)$ \\
\hline 41 & $-2.625830178824(-1)$ & 87 & $-1.357299424342(-2)$ & 133 & $-2.610501268195(-3)$ \\
\hline 42 & $-2.386362542385(-1)$ & 88 & $-1.298173461843(-2)$ & 134 & $-2.535474186705(-3)$ \\
\hline 43 & $-2.173691413760(-1)$ & 89 & $-1.242266195914(-2)$ & 135 & $-2.463104925729(-3)$ \\
\hline 44 & $-1.984292276456(-1)$ & 90 & $-1.189368166837(-2)$ & 136 & $-2.393279416921(-3)$ \\
\hline 45 & $-1.815170143332(-1)$ & 91 & $-1.139285694233(-2)$ & 137 & $-2.325889298771(-3)$ \\
\hline 46 & $-1.663770524355(-1)$ & 92 & $-1.091839530964(-2)$ & & \\
\hline
\end{tabular}


Table 13

Relativistic magnetizabilities $\chi$ (in the units of $\left.\alpha^{2} a_{0}^{3}\right)$ of hydrogenlike atoms in the excited state $3 d_{5 / 2}(\mu= \pm 3 / 2)$, obtained with $\alpha^{-1}=$ 137.035999139 (CODATA 2014).

\begin{tabular}{|c|c|c|c|c|c|}
\hline$Z$ & $\chi\left[\alpha^{2} a_{0}^{3}\right]$ & $Z$ & $\chi\left[\alpha^{2} a_{0}^{3}\right]$ & $Z$ & $\chi\left[\alpha^{2} a_{0}^{3}\right]$ \\
\hline 1 & $-4.867620421635(+5)$ & 47 & $-1.058323534299(-1)$ & 93 & $-7.918361781247(-3)$ \\
\hline 2 & $-3.042522620491(+4)$ & 48 & $-9.751733756547(-2)$ & 94 & $-7.611972956068(-3)$ \\
\hline 3 & $-6.010776626879(+3)$ & 49 & $-9.001516147331(-2)$ & 95 & $-7.320766554808(-3)$ \\
\hline 4 & $-1.902226145000(+3)$ & 50 & $-8.323177537031(-2)$ & 96 & $-7.043829849743(-3)$ \\
\hline 5 & $-7.793512994430(+2)$ & 51 & $-7.708561480069(-2)$ & 97 & $-6.780314089776(-3)$ \\
\hline 6 & $-3.759621088759(+2)$ & 52 & $-7.150573949303(-2)$ & 98 & $-6.529429401888(-3)$ \\
\hline 7 & $-2.030099377716(+2)$ & 53 & $-6.643027880940(-2)$ & 99 & $-6.290440146415(-3)$ \\
\hline 8 & $-1.190513756379(+2)$ & 54 & $-6.180512865865(-2)$ & 100 & $-6.062660681625(-3)$ \\
\hline 9 & $-7.435902767291(+1)$ & 55 & $-5.758285545965(-2)$ & 101 & $-5.845451497854(-3)$ \\
\hline 10 & $-4.881322592132(+1)$ & 56 & $-5.372177122068(-2)$ & 102 & $-5.638215685643(-3)$ \\
\hline 11 & $-3.335990893601(+1)$ & 57 & $-5.018515054602(-2)$ & 103 & $-5.440395706081(-3)$ \\
\hline 12 & $-2.356963901784(+1)$ & 58 & $-4.694056576690(-2)$ & 104 & $-5.251470434821(-3)$ \\
\hline 13 & $-1.712422639210(+1)$ & 59 & $-4.395932071410(-2)$ & 105 & $-5.070952454210(-3)$ \\
\hline 14 & $-1.274097654632(+1)$ & 60 & $-4.121596712729(-2)$ & 106 & $-4.898385570570(-3)$ \\
\hline 15 & $-9.676186616540(+0)$ & 61 & $-3.868789050846(-2)$ & 107 & $-4.733342535959(-3)$ \\
\hline 16 & $-7.481136176902(+0)$ & 62 & $-3.635495450741(-2)$ & 108 & $-4.575422955862(-3)$ \\
\hline 17 & $-5.875620054953(+0)$ & 63 & $-3.419919478496(-2)$ & 109 & $-4.424251366043(-3)$ \\
\hline 18 & $-4.679342304402(+0)$ & 64 & $-3.220455481723(-2)$ & 110 & $-4.279475463494(-3)$ \\
\hline 19 & $-3.773195304098(+0)$ & 65 & $-3.035665734857(-2)$ & 111 & $-4.140764477830(-3)$ \\
\hline 20 & $-3.076633873577(+0)$ & 66 & $-2.864260622431(-2)$ & 112 & $-4.007807670844(-3)$ \\
\hline 21 & $-2.534042150749(+0)$ & 67 & $-2.705081417875(-2)$ & 113 & $-3.880312953076(-3)$ \\
\hline 22 & $-2.106291347955(+0)$ & 68 & $-2.557085285304(-2)$ & 114 & $-3.758005607333(-3)$ \\
\hline 23 & $-1.765382214118(+0)$ & 69 & $-2.419332189726(-2)$ & 115 & $-3.640627110021(-3)$ \\
\hline 24 & $-1.490969094821(+0)$ & 70 & $-2.290973449453(-2)$ & 116 & $-3.527934042019(-3)$ \\
\hline 25 & $-1.268059116666(+0)$ & 71 & $-2.171241704762(-2)$ & 117 & $-3.419697081585(-3)$ \\
\hline 26 & $-1.085461165369(+0)$ & 72 & $-2.059442110622(-2)$ & 118 & $-3.315700072465(-3)$ \\
\hline 27 & $-9.347227108708(-1)$ & 73 & $-1.954944589623(-2)$ & 119 & $-3.215739161004(-3)$ \\
\hline 28 & $-8.093897766039(-1)$ & 74 & $-1.857177005044(-2)$ & 120 & $-3.119621996614(-3)$ \\
\hline 29 & $-7.044845061380(-1)$ & 75 & $-1.765619134132(-2)$ & 121 & $-3.027166990465(-3)$ \\
\hline 30 & $-6.161314956150(-1)$ & 76 & $-1.679797338603(-2)$ & 122 & $-2.938202627708(-3)$ \\
\hline 31 & $-5.412872725522(-1)$ & 77 & $-1.599279843812(-2)$ & 123 & $-2.852566828956(-3)$ \\
\hline 32 & $-4.775422297476(-1)$ & 78 & $-1.523672550248(-2)$ & 124 & $-2.770106357134(-3)$ \\
\hline 33 & $-4.229740768873(-1)$ & 79 & $-1.452615311429(-2)$ & 125 & $-2.690676266125(-3)$ \\
\hline 34 & $-3.760383401171(-1)$ & 80 & $-1.385778621 \quad 122(-2)$ & 126 & $-2.614139387965(-3)$ \\
\hline 35 & $-3.354857877930(-1)$ & 81 & $-1.322860660446(-2)$ & 127 & $-2.540365855597(-3)$ \\
\hline 36 & $-3.002996213716(-1)$ & 82 & $-1.263584661884(-2)$ & 128 & $-2.469232658469(-3)$ \\
\hline 37 & $-2.696473109242(-1)$ & 83 & $-1.207696552813(-2)$ & 129 & $-2.400623228467(-3)$ \\
\hline 38 & $-2.428433770570(-1)$ & 84 & $-1.154962845981(-2)$ & 130 & $-2.334427053903(-3)$ \\
\hline 39 & $-2.193204229849(-1)$ & 85 & $-1.105168748459(-2)$ & 131 & $-2.270539319440(-3)$ \\
\hline 40 & $-1.986064334668(-1)$ & 86 & $-1.058116464198(-2)$ & 132 & $-2.208860570048(-3)$ \\
\hline 41 & $-1.803068694444(-1)$ & 87 & $-1.013623668380(-2)$ & 133 & $-2.149296397197(-3)$ \\
\hline 42 & $-1.640904584106(-1)$ & 88 & $-9.715221344419(-3)$ & 134 & $-2.091757145669(-3)$ \\
\hline 43 & $-1.496778518423(-1)$ & 89 & $-9.316564969600(-3)$ & 135 & $-2.036157639485(-3)$ \\
\hline 44 & $-1.368325209431(-1)$ & 90 & $-8.938831355972(-3)$ & 136 & $-1.982416925581(-3)$ \\
\hline 45 & $-1.253534103716(-1)$ & 91 & $-8.580691670813(-3)$ & 137 & $-1.930458033936(-3)$ \\
\hline 46 & $-1.150689806355(-1)$ & 92 & $-8.240915336976(-3)$ & & \\
\hline
\end{tabular}


Table 14

Relativistic magnetizabilities $\chi$ (in the units of $\left.\alpha^{2} a_{0}^{3}\right)$ of hydrogenlike atoms in the excited state $3 d_{5 / 2}(\mu= \pm 5 / 2)$, obtained with $\alpha^{-1}=$ 137.035999139 (CODATA 2014).

\begin{tabular}{|c|c|c|c|c|c|}
\hline$Z$ & $\chi\left[\alpha^{2} a_{0}^{3}\right]$ & $Z$ & $\chi\left[\alpha^{2} a_{0}^{3}\right]$ & $Z$ & $\chi\left[\alpha^{2} a_{0}^{3}\right]$ \\
\hline 1 & $-2.699969190335(+1)$ & 47 & $-1.191568236683(-2)$ & 93 & $-2.817812444465(-3)$ \\
\hline 2 & $-6.749691904782(+0)$ & 48 & $-1.141175262133(-2)$ & 94 & $-2.751836555806(-3)$ \\
\hline 3 & -2.999691907 161(+0) & 49 & $-1.093836351152(-2)$ & 95 & $-2.687937007096(-3)$ \\
\hline 4 & $-1.687191910493(+0)$ & 50 & $-1.049309646613(-2)$ & 96 & $-2.626027780046(-3)$ \\
\hline 5 & $-1.079691914777(+0)$ & 51 & $-1.007376768019(-2)$ & 97 & $-2.566027267424(-3)$ \\
\hline 6 & $-7.496919200131(-1)$ & 52 & $-9.678401290602(-3)$ & 98 & $-2.507858004372(-3)$ \\
\hline 7 & $-5.507123343646(-1)$ & 53 & $-9.305206060602(-3)$ & 99 & $-2.451446418634(-3)$ \\
\hline 8 & $-4.215669333417(-1)$ & 54 & $-8.952555058160(-3)$ & 100 & $-2.396722598180(-3)$ \\
\hline 9 & $-3.330252747679(-1)$ & 55 & $-8.618967896857(-3)$ & 101 & $-2.343620074845(-3)$ \\
\hline 10 & $-2.696919504799(-1)$ & 56 & $-8.303095176532(-3)$ & 102 & $-2.292075622759(-3)$ \\
\hline 11 & $-2.228324563456(-1)$ & 57 & $-8.003704817696(-3)$ & 103 & $-2.242029070391(-3)$ \\
\hline 12 & $-1.871919714286(-1)$ & 58 & $-7.719670030807(-3)$ & 104 & $-2.193423125196(-3)$ \\
\hline 13 & $-1.594552969417(-1)$ & 59 & $-7.449958700643(-3)$ & 105 & $-2.146203209892(-3)$ \\
\hline 14 & $-1.374470982299(-1)$ & 60 & $-7.193623998687(-3)$ & 106 & $-2.100317309493(-3)$ \\
\hline 15 & $-1.196920099991(-1)$ & 61 & $-6.949796063827(-3)$ & 107 & $-2.055715828311(-3)$ \\
\hline 16 & $-1.051607747627(-1)$ & 62 & $-6.717674614644(-3)$ & 108 & $-2.012351456172(-3)$ \\
\hline 17 & $-9.311764601641(-2)$ & 63 & $-6.496522375957(-3)$ & 109 & $-1.970179043196(-3)$ \\
\hline 18 & $-8.302539048471(-2)$ & 64 & $-6.285659218637(-3)$ & 110 & $-1.929155482498(-3)$ \\
\hline 19 & $-7.448431854419(-2)$ & 65 & $-6.084456925604(-3)$ & 111 & $-1.889239600264(-3)$ \\
\hline 20 & $-6.719209335684(-2)$ & 66 & $-5.892334508681(-3)$ & 112 & $-1.850392052653(-3)$ \\
\hline 21 & $-6.091660268747(-2)$ & 67 & $-5.708754011037(-3)$ & 113 & $-1.812575229067(-3)$ \\
\hline 22 & $-5.547725734824(-2)$ & 68 & $-5.533216738503(-3)$ & 114 & $-1.775753161335(-3)$ \\
\hline 23 & $-5.073185236891(-2)$ & 69 & $-5.365259870427(-3)$ & 115 & $-1.739891438392(-3)$ \\
\hline 24 & $-4.656717722711(-2)$ & 70 & $-5.204453406978(-3)$ & 116 & $-1.704957126092(-3)$ \\
\hline 25 & $-4.289220058383(-2)$ & 71 & $-5.050397415285(-3)$ & 117 & $-1.670918691798(-3)$ \\
\hline 26 & $-3.963305329925(-2)$ & 72 & $-4.902719541426(-3)$ & 118 & $-1.637745933418(-3)$ \\
\hline 27 & $-3.672928720366(-2)$ & 73 & $-4.761072759338(-3)$ & 119 & $-1.605409912608(-3)$ \\
\hline 28 & $-3.413105190364(-2)$ & 74 & $-4.625133331216(-3)$ & 120 & $-1.573882891852(-3)$ \\
\hline 29 & $-3.179694091421(-2)$ & 75 & $-4.494598956989(-3)$ & 121 & $-1.543138275160(-3)$ \\
\hline 30 & $-2.969233171984(-2)$ & 76 & $-4.369187093107(-3)$ & 122 & $-1.513150552167(-3)$ \\
\hline 31 & $-2.778809443108(-2)$ & 77 & $-4.248633423154(-3)$ & 123 & $-1.483895245386(-3)$ \\
\hline 32 & $-2.605957837939(-2)$ & 78 & $-4.132690464838(-3)$ & 124 & $-1.455348860441(-3)$ \\
\hline 33 & $-2.448581032744(-2)$ & 79 & $-4.021126299608(-3)$ & 125 & $-1.427488839067(-3)$ \\
\hline 34 & $-2.304885525969(-2)$ & 80 & $-3.913723412736(-3)$ & 126 & $-1.400293514708(-3)$ \\
\hline 35 & $-2.173330314016(-2)$ & 81 & $-3.810277633011(-3)$ & 127 & $-1.373742070563(-3)$ \\
\hline 36 & $-2.052585404558(-2)$ & 82 & $-3.710597162389(-3)$ & 128 & $-1.347814499910(-3)$ \\
\hline 37 & $-1.941498069980(-2)$ & 83 & $-3.614501686982(-3)$ & 129 & $-1.322491568574(-3)$ \\
\hline 38 & $-1.839065233514(-2)$ & 84 & $-3.521821561684(-3)$ & 130 & $-1.297754779412(-3)$ \\
\hline 39 & $-1.744410746675(-2)$ & 85 & $-3.432397061550(-3)$ & 131 & $-1.273586338688(-3)$ \\
\hline 40 & $-1.656766592302(-2)$ & 86 & $-3.346077693748(-3)$ & 132 & $-1.249969124220(-3)$ \\
\hline 41 & $-1.575457256826(-2)$ & 87 & $-3.262721564551(-3)$ & 133 & $-1.226886655202(-3)$ \\
\hline 42 & $-1.499886675483(-2)$ & 88 & $-3.182194796394(-3)$ & 134 & $-1.204323063596(-3)$ \\
\hline 43 & $-1.429527277491(-2)$ & 89 & $-3.104370990521(-3)$ & 135 & $-1.182263066998(-3)$ \\
\hline 44 & $-1.363910753824(-2)$ & 90 & $-3.029130731184(-3)$ & 136 & $-1.160691942902(-3)$ \\
\hline 45 & $-1.302620244839(-2)$ & 91 & $-2.956361127784(-3)$ & 137 & $-1.139595504265(-3)$ \\
\hline 46 & $-1.245283703557(-2)$ & 92 & $-2.885955391642(-3)$ & & \\
\hline
\end{tabular}


Table 15

Relativistic magnetizabilities $\chi$ (in the units of $\alpha^{2} a_{0}^{3}$ ) for states $1 s_{1 / 2}, 2 s_{1 / 2}, 2 p_{1 / 2}$ and $2 p_{3 / 2}$ of selected hydrogenlike ions, obtained with $\alpha^{-1}=137.035999139$ (CODATA 2014) [the upper entries] and with $\alpha^{-1}=137.035999074$ (CODATA 2010) [the lower entries].

\begin{tabular}{|c|c|c|c|c|c|}
\hline$Z$ & $1 s_{1 / 2}$ & $2 s_{1 / 2}$ & $2 p_{1 / 2}$ & $2 p_{3 / 2}(\mu= \pm 1 / 2)$ & $2 p_{3 / 2}(\mu= \pm 3 / 2)$ \\
\hline 1 & $\begin{array}{l}-4.999644993669(-1) \\
-4.999644993668(-1)\end{array}$ & $\begin{array}{l}-6.999722649147(+0) \\
-6.999722649147(+0)\end{array}$ & $\begin{array}{llll}6.676 & 163 & 152 & 752(+4) \\
6.676 & 163 & 146 & 418(+4)\end{array}$ & 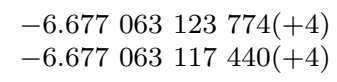 & $\begin{array}{l}-5.999861546894(+0) \\
-5.999861546894(+0)\end{array}$ \\
\hline 2 & $\begin{array}{l}-1.249645001764(-1) \\
-1.249645001764(-1)\end{array}$ & $\begin{array}{l}-1.749722649002(+0) \\
-1.749722649002(+0)\end{array}$ & $\begin{array}{l}4.171 \quad 164660 \quad 772(+3) \\
4.171 \quad 164656 \quad 814(+3)\end{array}$ & $\begin{array}{l}-4.173414370995(+3) \\
-4.173414367036(+3)\end{array}$ & $\begin{array}{l}-1.499861548141(+0) \\
-1.499861548 \quad 141(+0)\end{array}$ \\
\hline 5 & $\begin{array}{l}-1.996450584 \quad 458(-2) \\
-1.996450584 \quad 455(-2)\end{array}$ & $\begin{array}{l}-2.797226479764(-1) \\
-2.797226479762(-1)\end{array}$ & $\begin{array}{l}1.065244131032(+2) \\
1.065244130018(+2)\end{array}$ & $\begin{array}{l}-1.068841233198(+2) \\
-1.068841232184(+2)\end{array}$ & $\begin{array}{l}-2.398615568695(-1) \\
-2.398615568694(-1)\end{array}$ \\
\hline 7 & $\begin{array}{l}-1.016859395768(-2) \\
-1.016859395764(-2)\end{array}$ & $\begin{array}{l}-1.425797896419(-1) \\
-1.425797896416(-1)\end{array}$ & $\begin{array}{l}2.765269415 \quad 830(+1) \\
2.765269413 \quad 192(+1)\end{array}$ & $\begin{array}{l}-2.783607783733(+1) \\
-2.783607781095(+1)\end{array}$ & $\begin{array}{l}-1.223105464382(-1) \\
-1.223105464381(-1)\end{array}$ \\
\hline 10 & $\begin{array}{l}-4.964526105003(-3) \\
-4.964526104969(-3)\end{array}$ & $\begin{array}{l}-6.972264418235(-2) \\
-6.972264418208(-2)\end{array}$ & $\begin{array}{l}6.600466097095(+0) \\
6.600466090761(+0)\end{array}$ & $\begin{array}{l}-6.690176291 \quad 750(+0) \\
-6.690176285 \quad 416(+0)\end{array}$ & $\begin{array}{l}-5.986158805136(-2) \\
-5.986158805123(-2)\end{array}$ \\
\hline 20 & $\begin{array}{l}-1.214607414490(-3) \\
-1.214607414456(-3)\end{array}$ & $\begin{array}{l}-1.722262691678(-2) \\
-1.722262691651(-2)\end{array}$ & $\begin{array}{l}3.983443339545(-1) \\
3.983443335588(-1)\end{array}$ & $\begin{array}{l}-4.205544369778(-1) \\
-4.205544365818(-1)\end{array}$ & $\begin{array}{l}-1.486171290440(-2) \\
-1.486171290427(-2)\end{array}$ \\
\hline 40 & $\begin{array}{l}-2.774370778562(-4) \\
-2.774370778233(-4)\end{array}$ & $\begin{array}{l}-4.097522252752(-3) \\
-4.097522 \quad 252488(-3)\end{array}$ & $\begin{array}{llll}2.149 & 268 & 284 & 498(-2) \\
2.149 & 268 & 282 & 043(-2)\end{array}$ & $\begin{array}{l}-2.682735569862(-2) \\
-2.682735567380(-2)\end{array}$ & $\begin{array}{l}-3.612214346049(-3) \\
-3.612214345919(-3)\end{array}$ \\
\hline 50 & $\begin{array}{l}-1.651892129562(-4) \\
-1.651892129562(-4)\end{array}$ & $\begin{array}{l}-2.522402512753(-3) \\
-2.522402512490(-3)\end{array}$ & $\begin{array}{l}7.818803550 \quad 735(-3) \\
7.818 \quad 803540 \quad 792(-3)\end{array}$ & $\begin{array}{l}-1.112807953802(-2) \\
-1.112807952780(-2)\end{array}$ & $\begin{array}{l}-2.262592588983(-3) \\
-2.262592588 \quad 854(-3)\end{array}$ \\
\hline 80 & $\begin{array}{l}-4.446245639716(-5) \\
-4.446245636709(-5)\end{array}$ & $\begin{array}{l}-8.150967719944(-4) \\
-8.150967717275(-4)\end{array}$ & $\begin{array}{l}6.338207576 \quad 445(-4) \\
6.338207562 \quad 884(-4)\end{array}$ & $\begin{array}{l}-1.746639554569(-3) \\
-1.746639552928(-3)\end{array}$ & $\begin{array}{l}-8.017538525544(-4) \\
-8.017538524283(-4)\end{array}$ \\
\hline 100 & $\begin{array}{l}-1.749820929318(-5) \\
-1.749820926556(-5)\end{array}$ & $\begin{array}{l}-4.191628402646(-4) \\
-4.191628399901(-4)\end{array}$ & $\begin{array}{l}1.023054311643(-4) \\
1.023054307216(-4)\end{array}$ & $\begin{array}{l}-7.040609243754(-4) \\
-7.040609236324(-4)\end{array}$ & $\begin{array}{l}-4.658211084276(-4) \\
-4.658211083045(-4)\end{array}$ \\
\hline 120 & $\begin{array}{l}-3.838526853900(-6) \\
-3.838526830112(-6)\end{array}$ & $\begin{array}{l}-1.990375839852(-4) \\
-1.990375836816(-4)\end{array}$ & $\begin{array}{l}-8.557571732907(-6) \\
-8.557571841886(-6)\end{array}$ & $\begin{array}{l}-3.048757238 \quad 860(-4) \\
-3.048757234 \quad 136(-4)\end{array}$ & $\begin{array}{l}-2.844509502864(-4) \\
-2.844509501672(-4)\end{array}$ \\
\hline 130 & $\begin{array}{l}2.099852117594(-7) \\
2.099852324002(-7)\end{array}$ & $\begin{array}{l}-1.184030625960(-4) \\
-1.184030622281(-4)\end{array}$ & $\begin{array}{l}-1.633283429506(-5) \\
-1.633283430519(-5)\end{array}$ & $\begin{array}{l}-1.847126573331(-4) \\
-1.847126567976(-4)\end{array}$ & $\begin{array}{l}-2.239544235860(-4) \\
-2.239544234690(-4)\end{array}$ \\
\hline 137 & $\begin{array}{l}2.154582953028(-6) \\
2.154582969088(-6)\end{array}$ & $\begin{array}{l}-4.903302713912(-5) \\
-4.903302402152(-5)\end{array}$ & $\begin{array}{l}-9.398566990645(-6) \\
-9.398566418109(-6)\end{array}$ & $\begin{array}{l}-4.903236487356(-5) \\
-4.903235281066(-5)\end{array}$ & $\begin{array}{l}-1.894662493029(-4) \\
-1.894662491876(-4)\end{array}$ \\
\hline
\end{tabular}

